\title{
Gradhiva
}

GRADHIV

Revue d'anthropologie et d'histoire des arts

$21 \mid 2015$

Création plastique d'Haïti

\section{Le tatouage samoan et ses agents. Images, mémoire et actions rituelles}

Samoan tattooing and its agents: images, memory, and ritual actions

\section{Sébastien Galliot}

\section{(2) OpenEdition}

1 Journals

Édition électronique

URL : http://journals.openedition.org/gradhiva/2969

DOI : 10.4000/gradhiva.2969

ISSN : 1760-849X

Éditeur

Musée du quai Branly Jacques Chirac

Édition imprimée

Date de publication : 1 février 2015

Pagination : 156-181

ISBN : 978-2-35744-075-3

ISSN : 0764-8928

Référence électronique

Sébastien Galliot, "Le tatouage samoan et ses agents. Images, mémoire et actions rituelles », Gradhiva [En ligne], 21 | 2015, mis en ligne le 01 février 2015, consulté le 21 décembre 2020. URL http://journals.openedition.org/gradhiva/2969; DOI : https://doi.org/10.4000/gradhiva.2969 

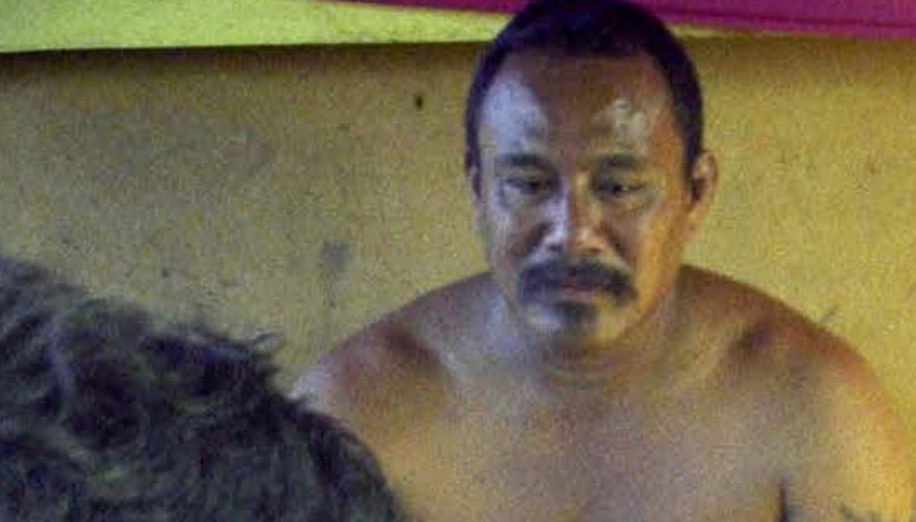

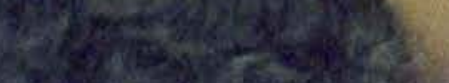

the
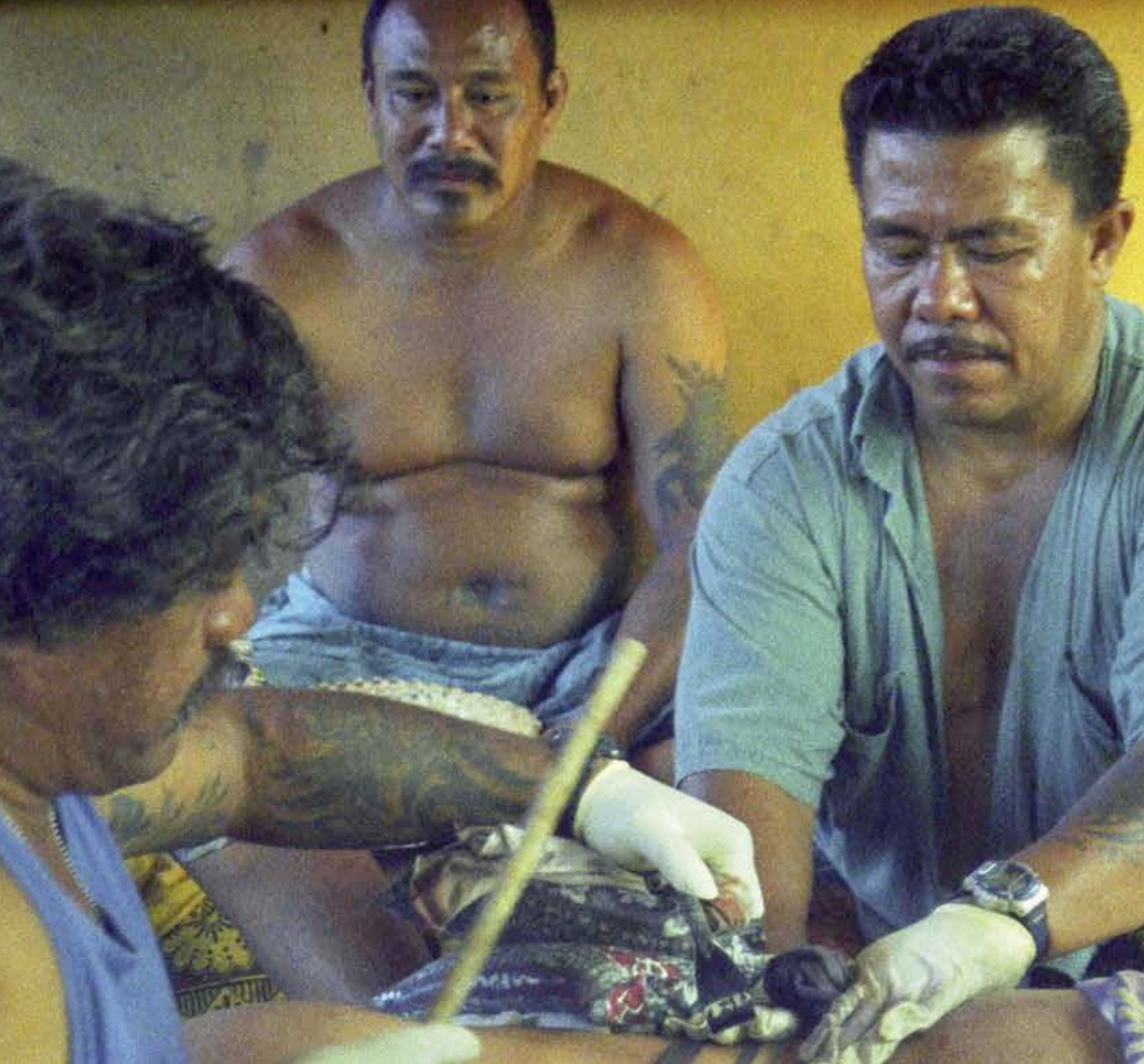

(6)
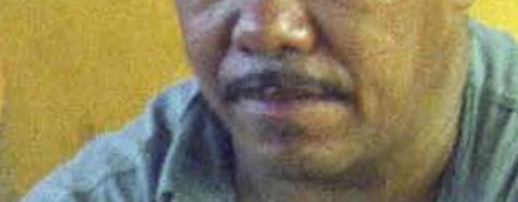

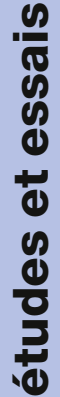
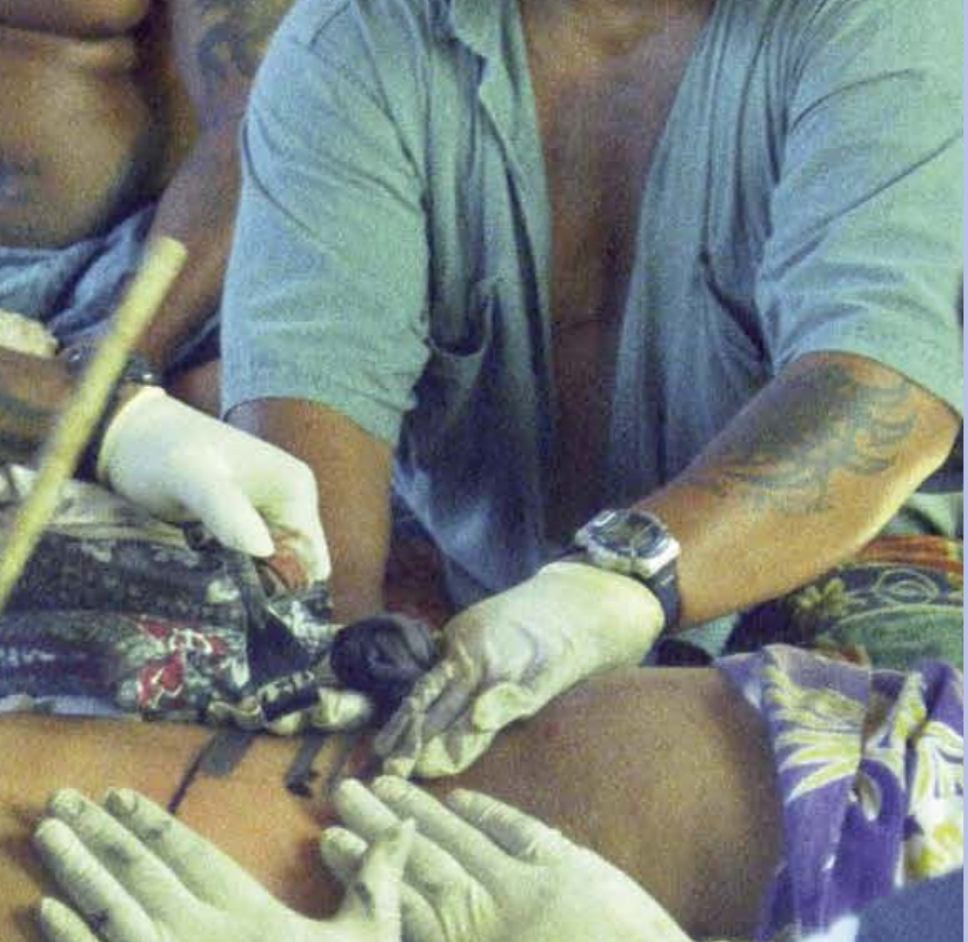

$\left.\ln ^{2}\right)^{2}$

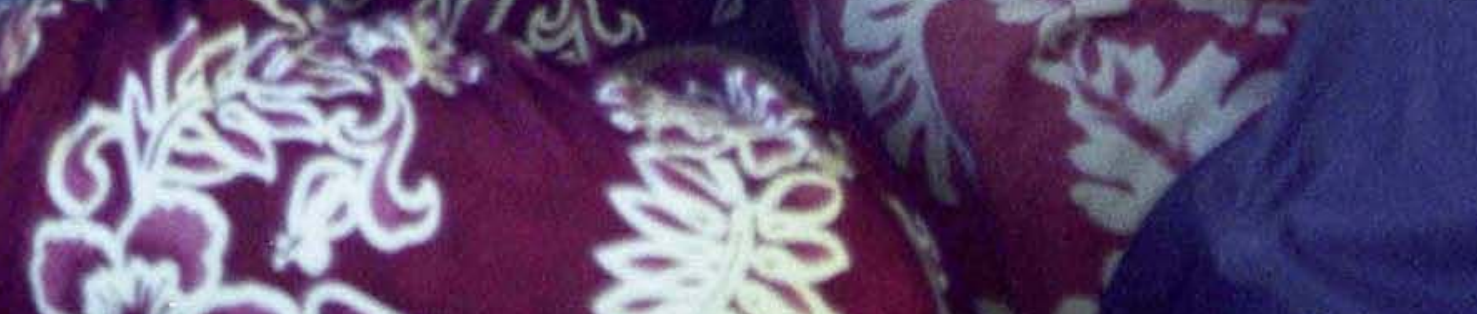

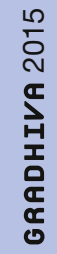




\section{Le tatouage samoan et ses agents. Images, mémoire et actions rituelles}

par Sébastien Galliot

Les Samoans produisent deux tatouages, l'un masculin appelé pe'a, l'autre féminin appelé malu, devenus si emblématiques de leur identité que leurs motifs se retrouvent sur une multitude de médias (maillots de rugby, pagnes, sculptures, châssis de réacteur d'avion). Ces motifs, librement disséminés et commercialisés, contrastent avec les œuvres rituelles produites et contrôlées par une communauté d'experts tatoueurs. Si la technologie du tatouage peut servir de ressource pour la production de sujets politiques, elle renvoie à une image rituelle complexe mobilisant des procédés figuratifs très élaborés dont la mémorisation et la transmission ne reposent pas strictement sur une articulation de l'image à la parole mais de l'image au corps. 


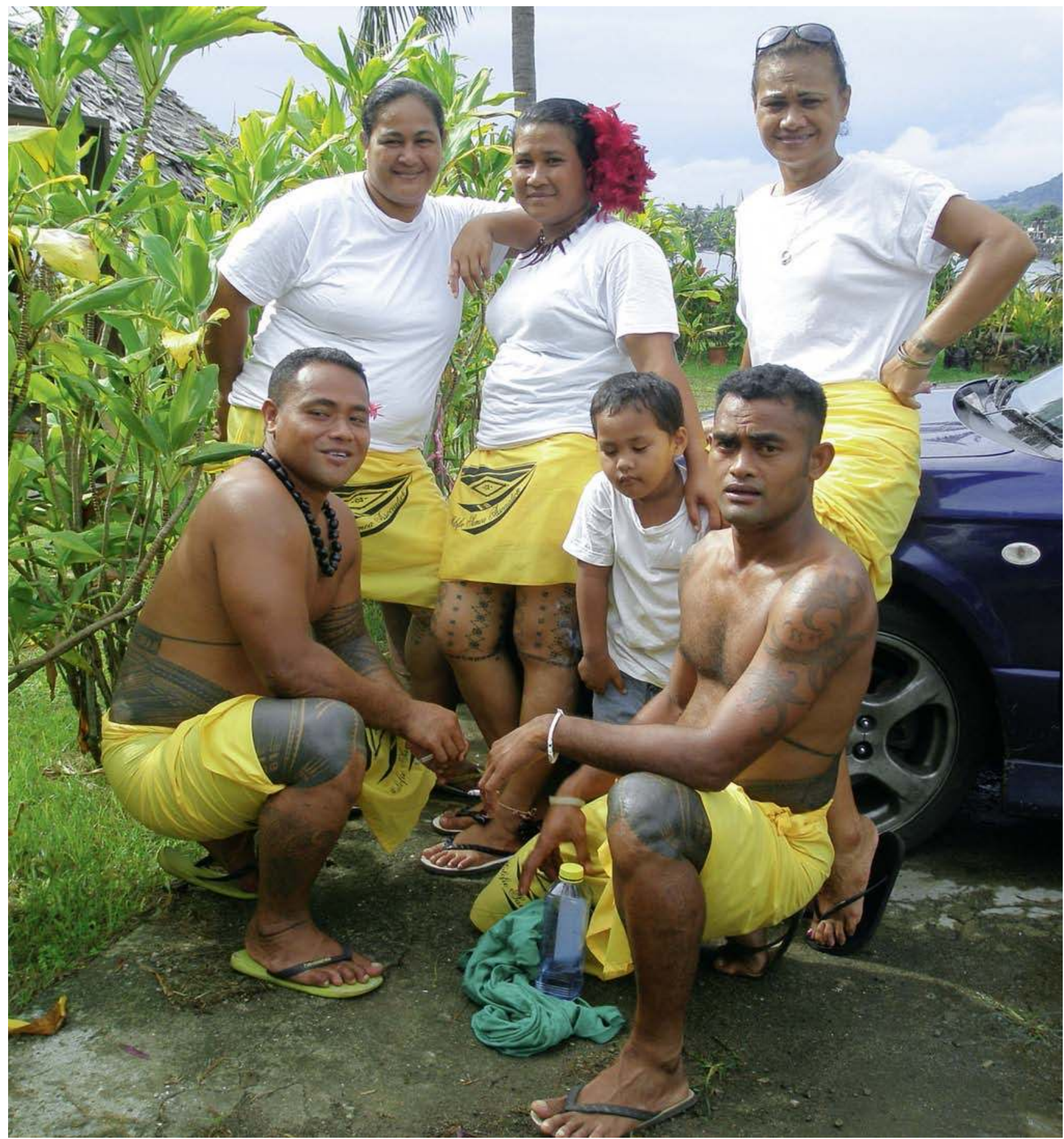

fig. 1

Jeunes membres de

l'association Malofie

qui regroupe plusieurs

centaines de porteurs

de tatouages rituels,

Apia, juin 2012. Photo

Sébastien Galliot. 
S'ils manifestent un intérêt pour le tatouage réalisé au dermographe et les compositions ornementales de type «néo-tribal polynésien ${ }^{\mathbf{1}}$ », nombreux sont les Samoans des îles aussi bien que de la diaspora qui restent attachés aux marques standardisées masculines (pe'a) et féminines (malu ${ }^{2}$, fig. 1) que permet d'obtenir une technique de tatouage par percussion effectuée dans le contexte d'un rituel et demeurant le privilège d'un corps de métier spécialisé ou d'experts. L'exceptionnelle continuité rituelle, technique et iconographique du tatouage samoan - qui a résisté dès le XIX siècle aux assauts du prosélytisme religieux des missionnaires tant protestants que catholiques $^{3}$-, sa popularité grandissante depuis les années 1970-1980 et l'adoption de son répertoire de motifs par un nombre croissant de tatoueurs occidentaux n'ont paradoxalement pas suscité d'enquête ethnographique approfondie sur les modalités de sa transmission. Témoignant d'une exceptionnelle résilience, le rituel et les images réalisées durant son déroulement permettent pourtant de s'interroger sur des aspects rarement traités dans la littérature sur le tatouage en Polynésie. Il est possible en effet d'élargir dans un tel contexte le débat sur le tatouage en Polynésie au-delà de l'analyse des conditions historiques de son abandon ou des dynamiques sociales de sa réappropriation ${ }^{4}$ et d'interroger le tatouage par de nouvelles questions. Quels rapports existe-t-il entre les cadres sociaux de la transmission du tatouage et les supports mnémoniques de son imagerie lorsque celle-ci n'est pas transmise d'une génération à l'autre à travers la préservation d'une culture matérielle mais par une forme spécifique de performance rituelle ${ }^{5}$ ? Si l'on tient compte du fait que les images produites, bien que hautement complexes, se réalisent via des activités essentiellement non verbales, quels sont les processus de mémorisation engagés pour maintenir cette tradition iconographique? Comment corps, image, mémoire et savoir-faire s'articulent-ils les uns les autres dans le rituel et quels liens ce complexe relationnel entretient-il par ailleurs à l'iconicité des motifs réalisés?

Reposant sur deux années d'enquête de terrain à Samoa et centré sur la fabrication d'images rituelles tatouées, cet article entend examiner ces différentes questions afin de contribuer à l'étude des mécanismes de transmission culturelle mobilisés au cours de l'action rituelle. II vise à expliquer plus particulièrement la relation entre la forme donnée aux tatouages, les procédés de leur reproduction et les connaissances qu'ils véhiculent, en tenant compte des questions spécifiques qui se posent aux agents - les maîtres tatoueurs et leurs apprentis - et aux patients d'un rituel qui engage leur corps dans une expérience physique douloureuse prolongée par une satisfaction esthétique. II entend ainsi contribuer aux travaux (encore trop rares) ayant attiré l'attention sur le fait que la transmissibilité des rituels - et leur efficacité - repose sur une interaction entre les corps, les lieux, les moments et les objets (Mitchell 2006) ou encore résulte de leur inextricable lien à des activités techniques (Balfet 1975; Lemonnier 2005, 2010 et 2012) comme c'est souvent le cas en Océanie. La réalisation des images tatouées à Samoa figure parmi ces pratiques rituelles au sein desquelles les gestes et les techniques ne sont pas seulement expressifs et symboliques mais poursuivent indubitablement des objectifs matériels. Le «sens» et le statut que l'on peut attribuer aux images produites doivent donc être à même d'en rendre compte ${ }^{6}$.
1. Cette appellation en vigueur chez la plupart des praticiens désigne toutes les formes de tatouages présentant des combinaisons de motifs empruntés aux corpus iconographiques maori, hawaiien, marquisien et samoan.

2. Ces deux types de tatouage sont regroupés sous le terme malofie, qui est d'usage formel et cérémoniel.

\section{Certains chefs}

samoans ont littéralement refusé le christianisme et ont continué d'organiser des opérations collectives de tatouage pendant la période d'installation des missionnaires (Galliot 2010).

\section{Ce champ de} recherche est aujourd'hui très populaire et a attiré l'attention de nombreux chercheurs. Voir à ce propos Thomas, Cole et Douglas (éd.) 2005, Kuwahara 2005, Te Awekotuku et Waimarie Nikora 2010 [2007] ou encore Mangos et Utanga 2011.

5. Voir à ce propos Küchler 2002 et Morphy 1991.

6. La perspective adoptée dans ce travail se nourrit et s'inscrit dans le renouveau de l'anthropologie de la culture matérielle et de celle des savoir-faire et des techniques. Elle est affine aux approches développées par Marie-Pierre Julien et Jean-Pierre Warnier (dir., 1999), qui en étudiant le couplage du corps et de l'environnement matériel ont permis de penser la continuité entre techniques d'objets et techniques du corps, et de donner plus de consistance à la notion d'incorporation dont les contours étaient jusquelà essentiellement phénoménologiques (Csordas [éd.] 1994; voir également la critique de Paul Connerton dans Olsen 2010). Elle s'inscrit aussi dans le vaste champ des travaux consacrés à l'évolution des mécanismes cognitifs responsables de l'acquisition et de l'évolution des compétences techniques (Leroi-Gourhan 1963 et 1964) ou de certains savoir-faire traditionnels (Chamoux 1978; Martinelli 1996; Mahias 2002) où l'ancrage matériel des questions de mémoire et de transmission a été souligné par quatre décennies d'ethnologie des techniques. 
7. Dans cet article, les expressions «maître tatoueur " et tufuga tā tatau seront employées indifféremment. Tufuga est un terme qui exprime l'expertise ou l'excellence technique.

8. Voir sur ce point en particulier Abercromby 1891, Fraser 1896 et Krämer 1994-1995 [1902-1903] (I : 151-152).

9. Le groupe de travail en question ici est appelé 'autufuga; il comprend le maître tatoueur et ses assistants.

10. Comparaison réalisée sur la base de l'ethnographie de Noel Lawrence McGrevy (1973) et de mes données mises à jour jusqu'en 2012. Cette apparente diminution du nombre de tufuga est contrebalancée par le fait que plusieurs d'entre eux résident et exercent dans la diaspora installée en banlieue des grandes métropoles de Nouvelle-

Zélande et d'Australie.

\section{Le tatoueur samoan : un maître titré}

Le «maître » ou «expert tatoueur » (tufuga tā tatau ou tufuga tā pe’a7), doté d'un «titre de tatoueur ( (suafa tā pe'a) conféré lui-même à la suite d'un long apprentissage auprès d'un maître, est le seul praticien, à Samoa, qui puisse exécuter des tatouages ayant la valeur d'actes rituels. Tous les tufuga se répartissent entre porteurs des titres su'a et tulou'ena, qui servent également d'éponymes à deux parentèles cognatiques ('aiga) et partagent une origine mythique commune. On rapporte en effet que les divinités tutélaires Taemā et Tilafaigā, des sœurs siamoises (reliées par le dos), transmirent le savoir-faire, le droit d'usage des outils à tatouer, des prérogatives cérémonielles ainsi que les titres qui leur correspondent aux chefs Tapu et Auva'a au cours d'un voyage qu'elles réalisèrent à travers l'archipel.

L'agrégation à l'un des deux groupes de maîtres tatoueurs requiert, en principe, trois rites d'habilitation. Deux ne sont pas spécifiques à l'art du tatouage mais néanmoins requis: tout maître tatoueur doit lui-même avoir été tatoué (porter un pe'a) ainsi qu'avoir été investi d'un «titre de chef» (suafa matai). Le troisième rite d'habilitation, appelé fafano, ponctue quant à lui l'apprentissage du «métier » proprement dit. II consiste en une cérémonie semi-privée au cours de laquelle un maître donne sa bénédiction (fa'amanuia) et une panoplie d'outils (fig. 2) à son apprenti. Les outils à tatouer (au tā tatau ou autā) et l'acte de les transmettre concrétisent un lien généalogique entre les divinités tutélaires et les descendants actuels des ancêtres rencontrés par ces dernières au cours de leur voyage mythique dans l'archipel ${ }^{8}$.

La transmission habituelle (et autrefois réputée la plus commune) des titres de tatoueur (suafa tā pe'a) se réalise en ligne agnatique aux fils et aux fils de frères selon un mode de transmission désigné par le terme tamatāne. Toutefois, à l'instar des titres de chef, la transmission de ceux de maitre tatoueur reste ouverte à d'autres modalités. Elle dépend alors du rang familial et des mérites propres du récipiendaire, qu'il s'agisse de son implication manifeste dans le groupe de travail ou de la générosité avec laquelle il s'acquitte de ses actes de service ${ }^{9}$. S'ajoute le fait que, pour être dûment reconnu, tout maître doit bien évidemment faire montre d'un haut niveau d'expertise technique, de la fabrication des outils à la mémorisation exacte des images à produire sans omettre les procédures thérapeutiques et rituelles qui entourent l'opération de tatouage elle-même.

L'ensemble des contraintes sociales et rituelles qui déterminent l'accession au statut de maître tatoueur restreignent le nombre d'individus capables de - et autorisés à - réaliser ce type d'images. À Samoa, on comptait en 1973 environ un maître tatoueur pour onze mille habitants. Cette proportion a diminué à l'heure actuelle et avoisine un pour trente mille ${ }^{\mathbf{1 0}}$. Cette proportion limitée de maîtres tatoueurs, qui peut être interprétée comme une stratégie de monopole des porteurs de titre, s'explique aussi par la nature des connaissances en jeu. En effet, si l'apprentissage des motifs ornementaux peut aujourd'hui se faire en consultant les nombreux sites internet dédiés au tatouage polynésien, et si certains tatoueurs peuvent trouver dans le monde digital une source d'inspiration (se passant de la relation maître-assistant qui prévaut à Samoa), la dimension surnaturelle 


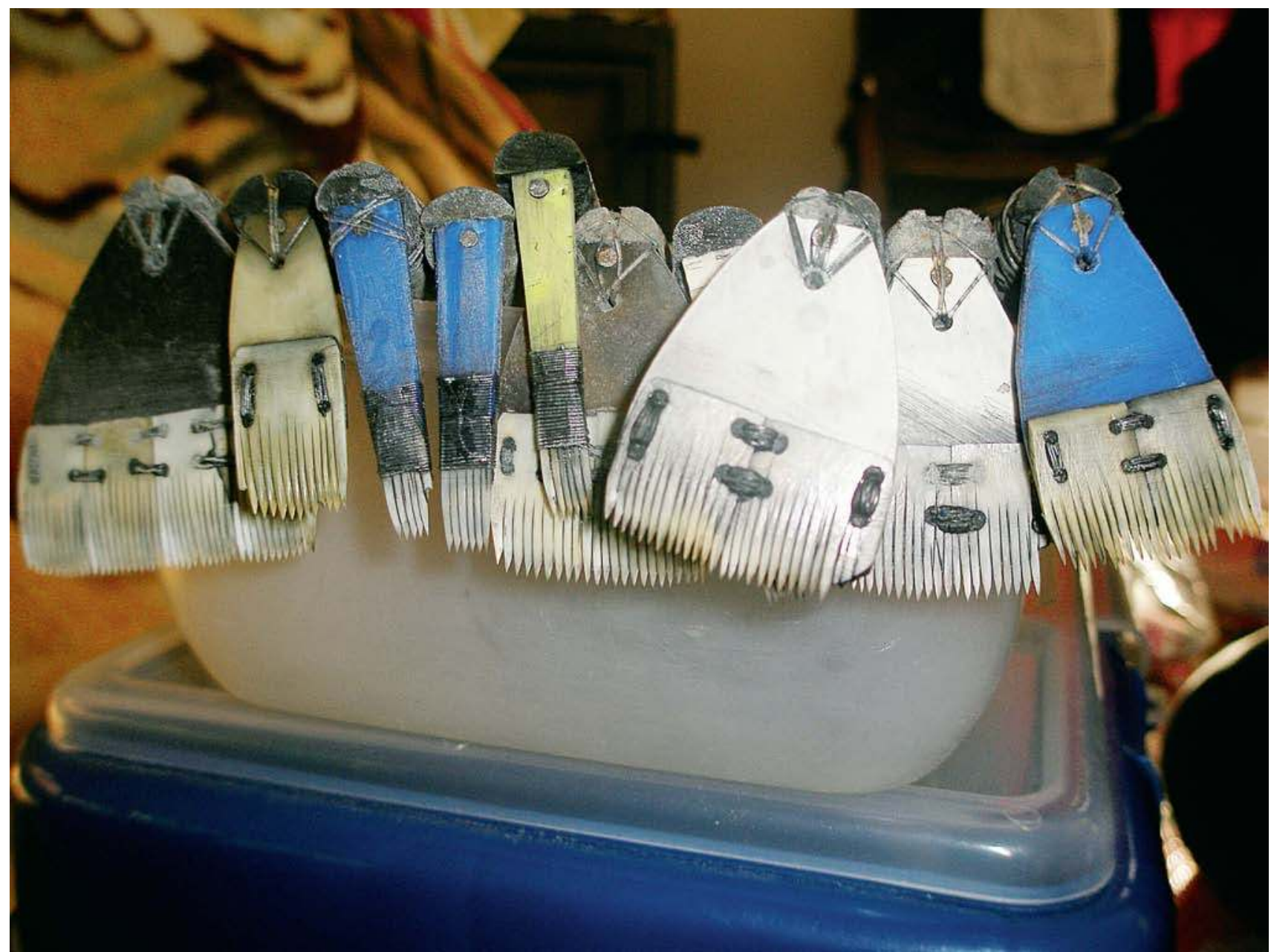

fig. 2

Panoplie d'outils

appartenant à Su'a

Fa'alavelave Vitale. Naenae,

Nouvelle-Zélande, 2007.

Photo Sébastien Galliot 
11. Ces titularisations ont pu être mal interprétées. En effet, tandis que le fa'asamoa (la manière samoane) consiste à mobiliser la famille étendue des impétrants pour réunir et présenter les dons obligatoires dans tout saofa'i (cérémonie de titularisation), les étrangers concernés ont essentiellement contribué à leur titularisation en versant des sommes d'argent, ce qui a pu être perçu comme un achat.

12. C'est notamment le cas du maître tatoueur Loli Tikeri (ancien apprenti de Sulu'ape Alaiva'a), porteur du titre loli qui lui confère des fonctions cérémonielles de premier plan dans son village d'origine; le contexte rituel du tatouage ne nécessite à son égard que le terme d'adresse su'a. de cette activité et la production de cette image rituelle demeurent très largement indissociables de l'autorité et du savoir-faire incarnés par les maîtres tatoueurs.

Les caractéristiques générales touchant cette "corporation " ne signifient pas que les modalités qui permettent de l'intégrer, ou du moins de s'y trouver rattaché, n'aient pas été affectées par les enjeux des processus sociaux contemporains touchant les îles et par la célébrité progressive de la pratique elle-même dans le monde du tatouage occidental. Quelques données historiques sont indispensables pour mieux préciser cet aspect. Dans les années 1960, se sont installés dans la banlieue d'Auckland, foyer de la diaspora samoane en Nouvelle-Zélande, deux maître tatoueurs cousins I'un de l'autre: Pasina Sefo et Sulu'ape Paulo II. Dans les années 1980, ce dernier a fortement contribué à faire (re)connaître le tatouage samoan par les relations qu'il a établies avec plusieurs artistes tahitiens et maoris qui souhaitaient être formés au maniement des outils traditionnels. Sulu'ape Paulo II et son frère Sulu'ape Alaiva'a ont été ainsi les premiers Samoans à être conviés en Europe à des conventions internationales de tatouage (en 1985 à Rome et en 1990 à Amsterdam), et le premier s'est également fait connaître pour avoir réalisé des tatouages rituels samoans sur des Occidentaux (Brunt 2005). Le réseau d'échanges de savoirs qu'il a su tisser bien au-delà de la diaspora a eu un rôle clé dans le processus de globalisation du tatouage samoan (Mallon 2005). Si ce rôle important a cessé après son assassinat en 1999 (lui-même indirectement lié à sa renommée internationale en tant que tatoueur), son frère a poursuivi dans la même voie.

C'est ainsi qu'en novembre 2001, à l'occasion d'une convention internationale organisée à Samoa pour commémorer son frère assassiné, Sulu'ape Alaiva'a a choisi de faire un geste envers six anciens amis et apprentis de son frère en leur décernant le titre de chef sulu'ape qui compose son nom et celui de son défunt frère. II a réitéré cet acte de reconnaissance à au moins six reprises à l'endroit de plusieurs personnes dont une femme, devenue Sulu'ape Angela Bolson ${ }^{11}$. II s'est gardé toutefois de transmettre le titre de tatoueur su'a (dont il est détenteur). Cette prudence témoigne des enjeux locaux de la transmission de ce titre, qui a également valeur de titre de chef dans plusieurs villages et dont l'usage par Sulu'ape Alaiva'a a été contesté dans le passé par certains. De fait, il importe de ne pas confondre le titre de suafa tā pe'a, dont le détenteur est reconnu publiquement comme ayant acquis tous les éléments qui entrent dans la composition du rituel de tatouage, et celui de suafa matai qui, fût-il le titre du plus célèbre des tatoueurs contemporains, n'a que peu d'importance dans le contexte rituel. D'ailleurs, les apprentis samoans qui ne sont pas issus de la famille Sulu'ape ne sont jamais investis de ce titre de chef et font uniquement usage des titres de maître tatoueur su'a ou tulou'ena comme terme d'adresse formel pendant le rituel ${ }^{\mathbf{1 2}}$.

Dans les cas précédents décrits par Sean Mallon (ibid.), comme dans d'autres cas plus récents qui m'ont été communiqués verbalement par Sulu'ape Alaiva'a lui-même, l'objectif de ces transmissions de titre est de célébrer un lien amical entre lui et ses récipiendaires ou entre ces derniers et son frère décédé. Par conséquent, la transmission du titre de sulu'ape à 
des personnes étrangères s'explique par la valeur de celui de su'a et peut être interprétée comme une habile stratégie propre à Sulu'ape Alaiva'a consistant à accroître la notoriété de son titre de matai dans le milieu du tatouage tout en s'assurant d'une forme de contrôle sur l'activité des personnes qui en sont porteuses. II s'agirait donc moins d'une transformation du système traditionnel qu'une stratégie visant à se faire une place dans I'histoire du tatouage occidental, à la manière d'artistes tatoueurs ayant marqué I'histoire contemporaine de cette pratique tels qu'Ed Hardy, Lyle Tuttle, Sailor Jerry ou la famille Leu, et de valoriser ainsi son nom dans un autre réseau que celui de la chefferie samoane ${ }^{\mathbf{1 3}}$.

Il est indéniable que la participation des maîtres tatoueurs samoans à un réseau de tatoueurs occidentaux et polynésiens a joué un rôle dans la diffusion de certains éléments technologiques et iconographiques et dans la transformation du mode de passation. Toutefois, les prérogatives rituelles, le système de transmission du savoir-faire et l'architecture de l'œuvre rituelle demeurent entre les mains d'une communauté d'experts samoans très réduite ${ }^{14}$. Le rôle prépondérant des maîtres tatoueurs formés et autorisés à produire des tatouages trouve d'ailleurs sa confirmation dans le fait que, dans la diaspora samoane ou ailleurs, la réalisation des tatouages pe'a et malu est généralement confiée à des tufuga que l'on a fait venir tout spécialement de l'archipel, à d'autres installés dans la diaspora mais dont l'apprentissage a été réalisé à Samoa ou encore à des praticiens émigrés tardivement et dotés d'une grande expérience du rite. Un seul tufuga, Kasala Sanele, se présente comme véritable autodidacte, mais ce statut inconfortable l'a récemment conduit à demander une autorisation verbale à Sulu'ape Alaiva'a pour poursuivre son activité. Étant lui-même originaire de Falealili, région traditionnellement associée au passage des divinités tutélaires du tatouage et à la création d'un lieu de culte aujourd'hui disparu, il n'a pas été jugé nécessaire de lui attribuer un titre.

\section{Initiation et apprentissage "par corps"}

À Samoa, l'exécution des tatouages se déroule dans un lieu appelé «maison du tatouage» (fale tā pe'a) ou «hutte sacrée» (apisā), placé sous la protection de Dieu par un prêtre ou un chef de famille. Ce lieu circonscrit un espace sacralisé, que les participants au rituel intègrent avec le devoir d'y maintenir un comportement approprié et de s'astreindre à diverses prohibitions. Avant que le rituel proprement dit ne débute, des représentants de la famille du sollicitant rencontrent le maître tatoueur qui leur rappelle que le candidat et sa famille devront se conformer à la «loi » des porteurs du titre et de leur «famille» ('äiga ${ }^{15}$ ). Ce formalisme, qui se justifie tout autant par le statut et la dignité du tatoueur que par la présence implicite de forces invisibles susceptibles de mettre le postulant en danger, contribue à solenniser la pratique.

Au cours du rituel à proprement parler, trois types d'activités et de «pôle d'agence» se trouvent plus particulièrement orchestrés, selon des modalités relationnelles que Michael Houseman (1993 et 1999) a déjà relevées dans d'autres contextes impliquant également d'infliger des souffrances intenses à un patient. L'agent principal, institué dans son rôle par sa maîtrise technique, est le tatoueur: comme on l'a vu, il est en effet le «maître» ou
13. Les bénéficiaires de la politique de transmission du titre de sulu'ape ne font pas tous commerce du tatouage. Certains de ceux qui exercent cette pratique n'utilisent pas systématiquement les outils traditionnels. Enfin, plus rares encore sont ceux qui réalisent des œuvres rituelles intégrales de type pe'a ou malu. Dans la grande majorité des cas, les dépositaires étrangers du titre se refusent d'ailleurs eux-mêmes à pratiquer le rituel et réalisent rarement des tatouages intégraux.

14. Les tatoueurs non samoans qui réalisent des pe'a ou des malu laissent souvent certaines parties non tatouées (comme le nombril) afin que le patient puisse aller accomplir la fin du rituel à Samoa s'il le souhaite.

15. L'expression consacrée en samoan est: o le tulāfono o le 'àiga sā Su'a ou o le tulāfono o le 'āiga sā Tulou'ena. 


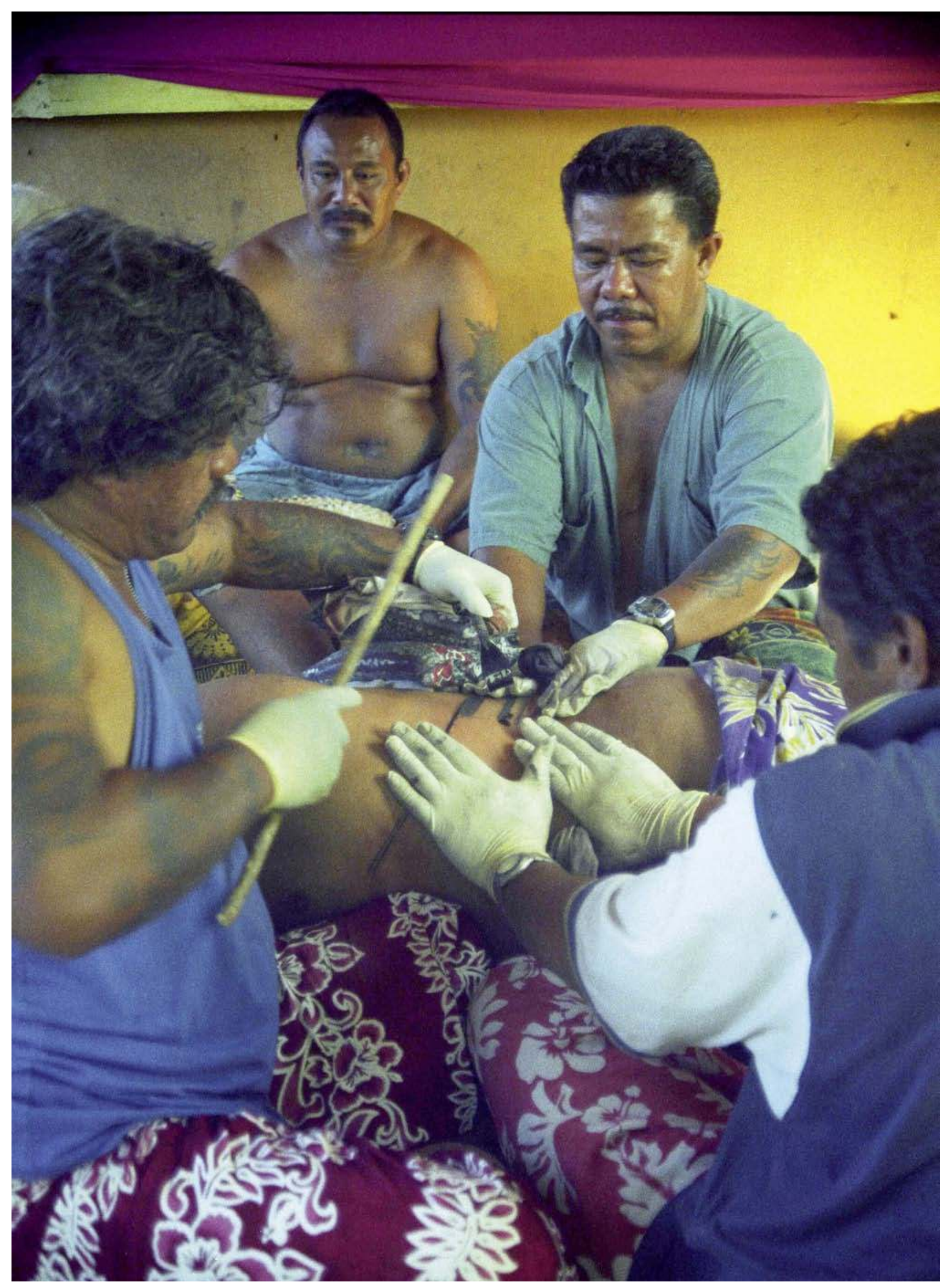


l'«expert» (tufuga). II n'agit pas seul; il est toujours accompagné de deux, voire trois assistants, eux-mêmes plus ou moins expérimentés dans cet office (fig. 3). Chargés plus particulièrement de tendre la peau du postulant, ces derniers constituent collectivement l'autufuga, «le groupe ('au-) du maître- (tufuga) ». Le maître et son groupe travaillent ensemble, assis autour du candidat qui se prête avec passivité et patience à leurs gestes et qui est désigné par la position spatiale qu'il doit adopter: il est le ta'oto (littéralement «celui qui est allongé, couché »). Immobile et immobilisé, car fortement maintenu par les assistants du tatoueur, il doit supporter la souffrance que génère la pénétration des instruments dans sa peau et n'est autorisé à changer de position qu'après de longs intervalles, en fonction du déplacement des zones à tatouer. Bien qu'il ne puisse voir l'exécution du tatouage, il remercie de temps à autre le tatoueur pour son travail. Parfois un parent lui tient la main, le pied ou la tête, ou chasse les mouches qui l'incommodent à l'aide d'un éventail. Les parents et les proches qui tiennent compagnie au postulant participent au rituel en arrière-plan: ils sont collectivement désignés comme le "groupe de la prière» ('autāpua'i) et leur fonction consiste à soutenir le patient en récitant des prières ou des encouragements (tāpua'i) - une série de phrases-types ${ }^{\mathbf{1 6}}$ - qu'ils énoncent épisodiquement afin que le rituel se déroule sans incidents.

Les actions du tatoueur et de ses assistants durant le processus de tatouage sont à la fois coopératives, hiérarchisées et spatialisées, et elles s'accompagnent d'une coordination visuelle dans laquelle le corps du patient tient lieu de médiation. Cette coordination visuelle assure le couplage fluide des gestes de percussion avec la position des mains des assistants pendant la mise en tension de la peau du patient. Le tufuga porte le regard sur les pointes de son outil et contrôle visuellement le positionnement des mains de l'assistant le moins expérimenté toujours assis à ses côtés, tandis que devant lui l'assistant le plus expérimenté regarde ses propres mains au moment de la mise en tension de la peau. Les déplacements des participants sont également contraints par des seuils spatiaux: les outils de l'expert et son environnement proche forment une zone considérée comme tapu («tabou», fa'asā en samoan) que nul n'est autorisé à franchir ou à occuper tandis que le lieu de l'opération est lui-même délimité par le cercle de personnes formé autour de l'action rituelle. Bien qu'on puisse admettre que la cartographie mentale de motifs à tatouer est relativement indépendante du corps de chaque patient singulier, elle n'en est pas moins ancrée dans des corps et cet ancrage concret facilite la coopération du groupe du tatoueur ${ }^{17}$. Au demeurant, le fonctionnement du groupe du tatoueur nécessite un haut niveau de concentration et un mode de coopération flexible pour pouvoir s'adapter, sans concertation préalable, aux corps différents sur lesquels il agit et aux réactions de ses patients. Du point de vue de ce groupe, le caractère routinier de la performance rituelle et des gestes techniques ne concerne pas pour autant la totalité des actions. Pour certains assistants en situation d'apprentissage, c'est en effet dans ce domaine d'action socialement et culturellement structuré que se réalise la transmission, malgré l'absence de toute instruction verbale explicite.

Outre le respect des règles imposées par le tufuga à l'intérieur de la maison du tatouage, le bon déroulement du rituel est également
16. Mālōle sisila (silasila)! ( Félicitations pour ce que nous sommes en train d'observer "); Mālō le sausau! ("Félicitations pour les percussions»: le geste de l'expert qui percute le sommet de la partie dentée) ; Mālōle a'ao solo! ("Félicitations pour les mains qui s'agitent »); Mälōle toso! ("Félicitations aux assistants qui tendent la peau "); Māàōle onosa'i! ("Félicitations pour la patience »); Mälōle ta’oto! (« Félicitations à celui qui est allongé»). Ce à quoi les assistants et le tufuga répondent invariablement: Mālōle tāpua'i! ("Félicitations pour le tāpua'i»).

17. L'attention à l'ancrage matériel des processus coopératifs et de la "cognition située" a été traitée avec particulièrement de finesse par Edwin Hutchins (2005).

fig. 3

Tatouage de George Levave, Savalalo, Apia, 2001. Photo Sébastien Galliot. 
18. Cette «altération pigmentaire" est désignée par une expression constituée des termes aeva, employé à Wallis et Futuna pour désigner la mort consécutive à l'infraction d'un tapu (Grand 2007: 214 ; voir également «Samoan tattoo customers risk death - and an ancient curse", Sunday Star Times, 24 juin 2007), et lama, nom de la noix qui sert à produire le pigment et de l'arbre qui la produit, le noyer des Moluques ou noyer de bancoule (Aleurites moluccana). Les croyances relatives à ces phénomènes ont récemment connu un traitement paroxystique dans le long métrage The Tattooist, réalisé en 2007 par Peter Burger. étroitement lié à la qualité des relations entre les participants. Qu'elle reçoive le tatoueur ou qu'elle se déplace chez lui pour l'opération, la famille du patient est tenue de «prendre soin du tatoueur » (tausi tufuga). Ce traitement consiste à instaurer avec l'expert rituel une relation de respect (fa'aaloalo) similaire à celle qui régit les rapports hiérarchiques formels. Cela implique l'usage de termes d'adresse spécifiques et appropriés à sa fonction de tatoueur et la présentation (lafo) de dons quotidiens de nourriture, de cigarettes et d'argent aussi longtemps que dure l'opération. Cet encadrement du rituel est supervisé par un proche parent du patient appelé taufalemau: à la fois «commanditaire» de l'opération, maître de cérémonie et intermédiaire entre le tufuga et les autres membres de la famille, le taufalemau est également tatoué et détenteur d'un titre de chef, connaissant de ce fait l'ensemble des termes d'adresse prescrits par le contexte spécifique du rite de tatouage. Ce dispositif de médiation est crucial car une relation mal engagée avec le tatoueur ou le non-respect des «lois» (tulāfono) explicitement rappelées (voir ci-dessus) peuvent entraîner des conséquences néfastes pour le patient.

Si l'apparence que prend l'œuvre tatouée pendant l'opération et la cicatrisation n'est pas conforme aux attentes ou présente des anomalies, celles-ci sont généralement imputées à la transgression d'un interdit ou à une mauvaise gestion des relations entre le groupe du spécialiste et la famille du patient. La principale conséquence du non-respect des règles imposées par le tatoueur est le lama'avea, une altération du pigment du tatouage perçue comme une malédiction pouvant entraîner la mort ${ }^{\mathbf{1 8}}$. Un tatouage réalisé dans son intégralité sans défauts visibles atteste en revanche la bonne santé physique du patient, sa vigueur sexuelle potentielle mais également les bons rapports entretenus entre les participants durant le rituel.

En ce qu'il implique une mise à l'épreuve par une douleur consentie et une invisibilité des opérations mêmes dont son corps est l'objet, le rituel du tatouage a une franche dimension initiatique pour le patient. Néanmoins, il présente en définitive une dimension révélatoire limitée: importe pour ce dernier l'expérience de la douleur corporelle intense, inédite et unique. Le tatouage en tant qu'image ne véhicule pas un savoir auquel seuls les initiés pourraient accéder. Ainsi, du point de vue des patients, la motivation pour ce type de rituel physiquement très éprouvant peut être reconduite et transmise dans la mesure où la réponse affective et émotive engendrée par cette initiation confirme et renforce les dispositions du porteur et les représentations des participants relatives à sa personne (son courage, sa résignation, son endurance), l'appartenance à une classe d'âge et ses rapports avec les autres groupes sociaux. En dépit du caractère inscrit de l'image rituelle, la mémoire du rituel partagée par les patients repose avant tout sur le souvenir d'une expérience physique traumatique et sur la croyance en l'intervention possible d'agents immatériels ou surnaturels mais s'exprimant à travers les gestes du tufuga, ses outils et le pigment. Elle s'inscrit globalement dans un contexte relationnel et multisensoriel dont le patient mémorise d'infimes détails: les personnes présentes pendant le rituel, les amitiés ou inimitiés qui se nouent en périphérie de l'opération, l'odeur du sang, les sensations diverses provoquées en fonction des différents types d'outils utilisés et des zones du corps concernées, les massages 
quotidiens des plaies par les assistants, les vives émotions exprimées lors de la cérémonie de clôture ${ }^{\mathbf{1 9}}$ ou encore les odeurs florales de ce moment, contrastant avec les odeurs carnées du tatouage. On peut donc reprendre et appliquer au tatouage samoan ce que de nombreux anthropologues ont déjà mis en évidence en étudiant les phénomènes de transmission au sens large des actions rituelles douloureuses et uniques, infligées sur le corps dans un cadre initiatique et volontairement subies (voir Barth 1975, Whitehouse 2000 ou encore McCauley et Lawson 2002): il mobilise chez les patients un certain type de mémoire (épisodique) et de restitution de l'expérience vécue qui relève de données contextuelles et biographiques. En dehors du rituel, le corps porteur de l'œuvre est éventuellement à son tour un vecteur de la transmission dans la mesure où les appréciations esthétiques relatives à ces images peuvent jouer un rôle dans la motivation et le passage à l'acte d'autres candidats. Mais, pour l'essentiel, l'efficacité du rituel et la pérennisation de l'œuvre sont principalement garanties par les connaissances procédurales et le mode opératoire de l'expert ainsi que par la douleur initiatique infligée au patient.

Pendant le rituel, la transmission entre agents d'une part et des agents aux patients d'autre part résulte d'un acte d'incorporation, produit par ce que l'on peut appeler un apprentissage «par corps» pour reprendre l'expression particulièrement parlante forgée par Sylvia Faure (2000), qui mobilise de façon différentielle la saisie sensori-motrice des gestes dans un contexte spécifique d'interactions sociales. Les postulants aux tatouages expérimentent pour leur part dans leur chair une douleur intense, prolongée et potentiellement mortelle; les apprentis accompagnant le tufuga apprennent de leur côté dans l'action, par l'exercice du regard, par paliers d'insertion (Martinelli 1996) et sans pratique d'énonciation, le maniement des outils, les propriétés physiques de la peau humaine et le plan de construction de l'œuvre. L'intégration des apprentis au groupe du tatoueur par «participation périphérique légitime» (Lave et Wenger 1991) est double puisqu'elle concerne l'acquisition progressive et simultanée de compétences techniques et culturelles. Autrement dit, le contexte rituel de l'action permet d'inculquer la hiérarchisation des relations au sein du groupe de travail et avec le groupe du patient, les termes d'adresse cérémoniels, les gestes techniques et l'architecture de l'œuvre à réaliser. En revanche, selon que l'on se place du point de vue des agents ou des patients, ce sont des connaissances distinctes qui sont ainsi incorporées. Si l'on considère les actions respectivement infligées et subies, l'incorporation désigne des processus de codification distincts mais qui partagent néanmoins un même rapport général au corps. Dans un cas comme dans l'autre, celui-ci ne se réduit pas à une surface d'inscription (telle une feuille de papier) mais intervient comme une entité sensori-motrice par laquelle passent différents types d'expériences physiques.

\section{Mémorisation, fabrication et iconicité des images tatouées}

S'il est incontestable que la mémorisation des images tatouées et des formes qui les constituent peut se réaliser de différentes manières, l'association historique de ces images au rituel (et le fait que celui-ci constitue toujours le contexte principal de leur transmission) est un fait que l'on ne saurait tenir pour anodin. De ce point de vue, on peut constater que
19. La cérémonie de clôture du rituel, appelée samaga pe'a (" onction du tatouage»), consiste en un discours formel de reconnaissance du courage du ou des soga'imiti (non plus postulants mais récemment tatoués) et de louanges pour le travail du groupe du tatoueur, lequel procède à la levée des interdits rituels par une série d'actes sur leurs corps (onction avec le sama, mélange de poudre de curcuma et d'huile de noix de coco, application de jaune d'œuf sur le sommet du crâne, brèves incantations, distribution de colliers de fleurs ou de fruits parfumés). Cet événement, qui comprend également la rétribution du tatoueur par une série de dons, contraste avec l'opération proprement dite en ce qu'il permet une expression des émotions. Aux pleurs de joie et de fierté de la famille du soga'imiti répondent les discours emphatiques du tatoueur ou d'un orateur convoqué pour l'occasion. 


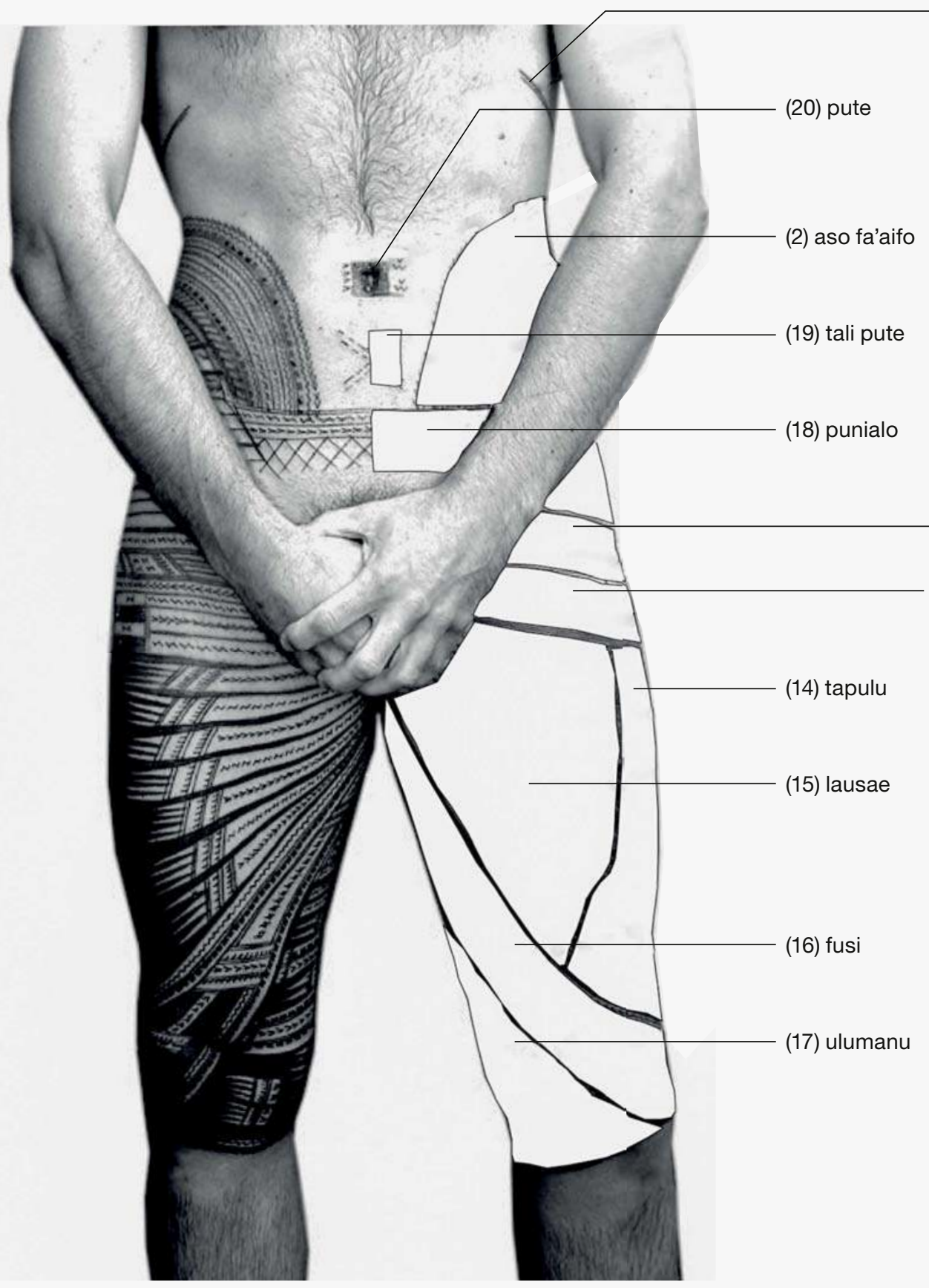

(1) fa'aulutao

(1) va'a

(2) aso e tasi

(3) pe'a / pulatama

(4) pulatele

(5) aso laiti

(6) tafani tapulu

(7) tafani teu

(8) saemutu

(9) fusi / selu

(10) aso tali'itu

(11) fa'apefu

(12) atigivae

(13) fa'amuli'ali'ao

(21) signature du tufuga

a. Pe'a vu de face 

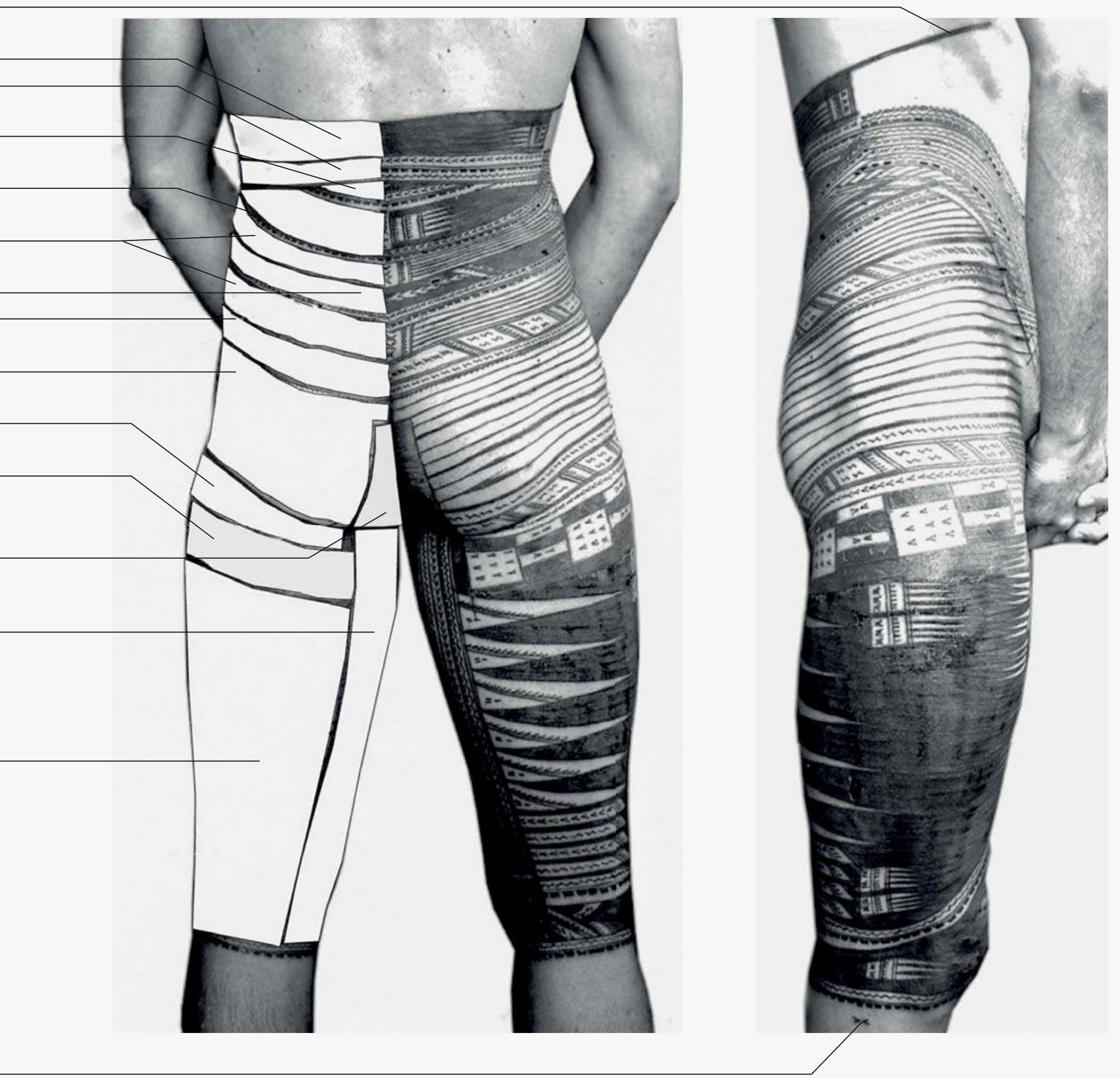

b. Pe'a vu de dos 
double page précédente

fig. 4

Sections vāega du tatouage masculin par ordre de réalisation de 1 à 20. Photo et schéma Sébastien Galliot. la procédure de «mise en dessin» des tatouages est fondamentale pour comprendre comment ces assemblages de formes sont mémorisables, et partant reproductibles, mais aussi à l'inverse combien la signification des motifs et les rapports iconiques sont dotés d'un «rendement » relativement faible pour les caractériser et par conséquent impropre à véhiculer des représentations stockées en mémoire explicite ou sémantique.

Comme on l'a déjà indiqué, le tatouage masculin pe'a est une image standardisée qui forme un tout, mais il se présente également comme une image complexe constituée à deux niveaux. En premier lieu, il résulte d'un assemblage de formes intermédiaires appelées vāega (fig. 4). Non seulement explicitement désignées, celles-ci sont également associées à un plan de construction ('ava'ega) qui dicte l'ordre de leur exécution et leur position relative. Tatouer un pe'a consiste ainsi à reproduire deux fois et en miroir une série vāega qui redouble la symétrie bilatérale du corps. C'est par la délimitation de ces formes intermédiaires par des pointillés, en partant du milieu du dos et en descendant jusque sur les cuisses, que les maîtres tatoueurs commencent par tatouer leur patient, morcelant son corps en portions invariables et produisant ainsi un quadrillage servant de guide aux assistants pour lui tendre la peau. Dans une seconde phase, le tatoueur complète ce canevas en procédant à son «remplissage»: les vāega sont ainsi achevées suivant les cas soit par la formation de larges aplats noirs, soit par l'adjonction d'ornements ou motifs décoratifs (mamanu). Les ornements sont eux-mêmes réalisés par l'application de règles récursives de production: un jeu de translation horizontale, verticale ou encore oblique où les motifs (ou séquences de motifs) sont répétés à l'intérieur des lignes qui délimitent les sections.

Le tatouage féminin, appelé globalement malu, partage ces caractéristiques bien qu'à un degré moindre. II ne comprend pas de formes intermédiaires mais consiste aussi en un assemblage (dans ce cas seulement de motifs ornementaux) produit par l'utilisation de règles récursives. Par le passé, certains maîtres tatoueurs réalisaient le malu en appliquant le procédé de la translation horizontale, et chaque motif ou séquence de motifs était répété horizontalement sur le pourtour de la cuisse de manière à former une ligne se superposant à la précédente. Actuellement, la majorité des tatoueurs assemblent le malu par translation verticale ou oblique et considèrent chaque face de la cuisse (antérieure, postérieure, externe et interne) à la manière d'une zone dans laquelle les mêmes motifs se répètent.

La dépendance intrinsèque des formes dessinées à leur procédure d'exécution va au-delà du simple séquençage et se manifeste aussi dans l'outillage utilisé. Le contour des vāega et le tatouage des lignes sont réalisés à l'aide d'une même catégorie d'outils (au sogi aso). L'instrument au tapulu permet d'obtenir de larges aplats noirs. Quant aux zones ornées de motifs décoratifs, elles requièrent l'usage de l'outil au mono (aiguille à remplissage). De taille variable, ces outils nécessitent différents types de percussions. Le son produit par leur maniement joue un rôle important dans l'apprentissage puisqu'il communique des informations sur leur «monnayage rythmique», pour reprendre l'expression appliquée par les musicologues à la subdivision du temps et à la densification rythmique. 
L'ensemble des aspects pratiques gouvernant la fabrication des motifs, fondamentaux pour leur mémorisation et leur reproduction, contraste avec le rôle mineur apporté par les informations linguistiques de la terminologie qui leur est associée ou les rapports iconiques des motifs eux-mêmes. Les termes pe'a et malu ont pour particularité de désigner par suite d'une synecdoque le motif complet: au sens strict ils servent de nom à un motif particulier - un triangle isocèle dans le milieu du dos des hommes (pe'a) et un losange dans le creux poplité des femmes (malu). Ils associent les motifs et le tatouage, et prennent leur nom respectivement à la roussette géante de Samoa - une grande chauve-souris frugivore (Pteropus samoensis) également appelée pe'a - et à la notion d'abri ou de protection dans le cas de malu, sans que personne puisse expliquer clairement pourquoi ils porteraient ces noms plutôt que d'autres. Dans les deux cas, les pièces intégrales signalant un rituel accompli se présentent comme des formes abstraites, aniconiques en quelque sorte puisque leur ressemblance avec la chose qu'elles désignent est loin d'être évidente. Néanmoins, elles représentent des formes reconnaissables fixées par la tradition du tatouage elle-même. Et dans la mesure où ces formes sont des entités à part entière et qu'elles ne dénotent (malgré leur nom) pas autre chose qu'elles-mêmes, elles constituent ainsi de véritables topoi picturaux. Pe'a et malu peuvent donc être considérés comme des «prototypes» (Gell 2009 [1998]: 32-33) dont chaque nouvelle occurrence est une réplique.

Le degré d'iconicité des formes intermédiaires vāega est quant à lui variable. Les vāega sont désignées soit par des termes vernaculaires dont le sens a été perdu - c'est le cas des zones appelées lausae, tafani et aso tali'itu -, soit elles évoquent indirectement des éléments de faune ou de flore locale: les zones fa'amuli'ali'ao présentent une forme triangulaire qui renvoie à des coquillages cylindriques appelés eux-mêmes ali'ao (Trochus spp.); les formes ulumanu, également triangulaires, rappellent vaguement un bec d'oiseau (ulumanu signifie littéralement "tête d'animal» ou "tête d'oiseau "). Certaines vāega peuvent figurer des éléments de culture matérielle comme la pirogue à double proue (va'a taumualua, fig. 4, section 1). À la différence des œuvres intégrales pe'a et malu, qui ont la propriété d'être reconnues et utilisées comme des emblèmes ethniques univoques, le système de correspondance entre les caractéristiques formelles des vāega et leur désignation respective reste fluctuant. Le corpus doctrinal devant être répété dans chaque tatouage n'est donc pas terminologique mais iconique. S'il n'est pas secret, il ne fait l'objet d'aucune communication ou exégèse verbale et sa mémorisation par les apprentis au fil des performances rituelles passe essentiellement par des inférences visuelles.

Les motifs ornementaux (mamanu) représentent divers éléments de culture matérielle, de faune ou de flore (fig. 5). La plupart sont exclusivement masculins ou féminins bien que certains soient mixtes. Ils sont toutefois sujets à des interprétations diverses. Lorsqu'il est exécuté par translation horizontale, le fa'asigano ("comme une fleur de pandanus") peut, en raison de sa forme, être appelé fa'aulutao ("comme une pointe de lance») ou encore fa'alautalo ("comme une feuille de taro»). Le répertoire iconographique des motifs décoratifs est composé de pictogrammes ou, pour utiliser la terminologie de Charles Sanders Pierce, de «signes iconiques» 
fig. 5

Répertoire ornemental du tatouage samoan.

Aso

Lignes généralement parallèles au nombre variable.

Elles peuvent être vierges ou remplies de plus petits motifs. Elles peuvent être droites ou courbes.

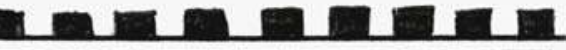

\section{Aso fa'atala}

Généralement utilisé pour marquer la limite d'une zone.

Ce motif est aussi utilisé comme une ligne décorative.

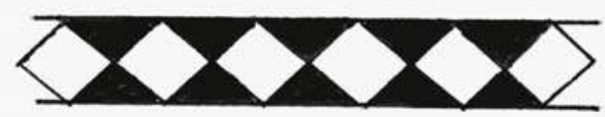

Fa'ataimane

Comme un diamant.

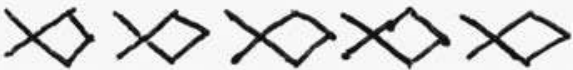

I'a

Poisson.

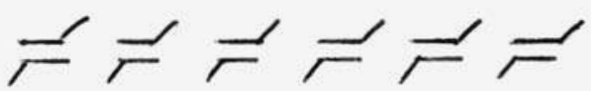

Fa'atigi pusa

Comme une boîte vide.

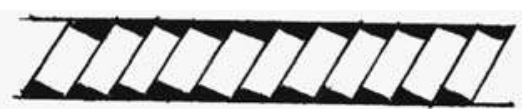

Fa'apola

Comme un store tressé de feuilles de cocotier.

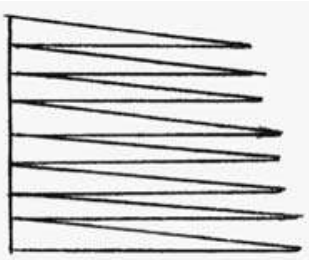

Fa'asigano

Comme une fleur de pandanus.

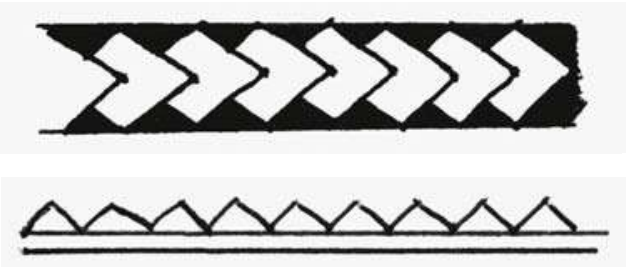

Fa'atala laupaogo

Comme les bords de la feuille de pandanus.

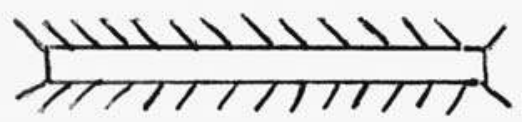

Fa'aatualoa

Représente le scolopendre. Ce motif est utilisé pour remplir les aso mais peut également être positionné à la verticale. Il est considéré comme un animal indésirable. Sa piqûre rappelle celle des instruments du tatoueur et peut provoquer le même type de nécrose que le tatouage. Sa grande fécondité est parfois invoquée pour expliquer sa présence dans le tatouage.
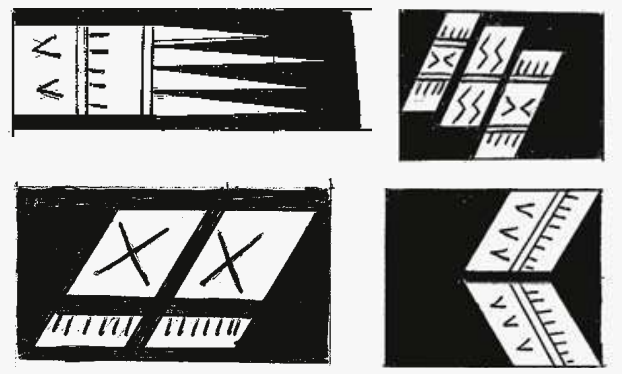

Fa'aila

De diverses formes, les fa'aila sont des décorations réalisées dans les aplats noirs tapulu.

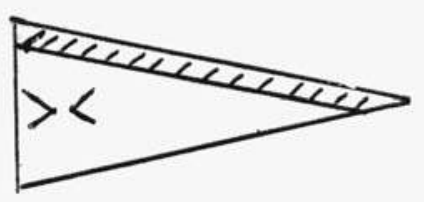

Fa'amuli'ali'ao

Ce motif qui évoque le coquillage ali'ao (Trochus) est presque exclusivement représenté à l'arrière de la jambe en séries verticales. 


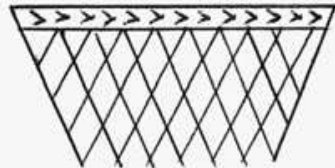

Fa'a'upega

Filet de pêche. Motif réservé à la zone pubienne punialo. Systématiquement surmonté de plusieurs aso, souvent orné de fa'avae'ali, parfois du prénom de la compagne du porteur.

Roussette (Pteropus samoensis).

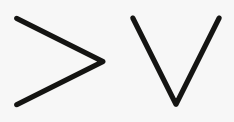

\section{Aso fa'avae'ali / fa'avaetūlī}

Ce motif consiste en une ligne ornée à intervalles réguliers du avae'ali en forme de $V$ et qui représente les pieds de l'appuie-tête samoan en bois.

\section{Vae tūlī}

Le tūlī (pluvier) est dans la mythologie samoane l'oiseau de la création. Le $V$ représenterait l'empreinte de ses pattes.

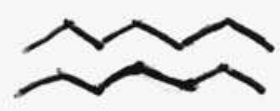

\section{Fa'a'anufe}

Chenille, ver. Très utilisé pour décorer les lignes et pour les petits intervalles des fa'aila.

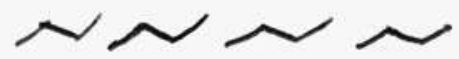

Fa'anufe pi'o tolu

Représentation d'une chenille en trois sections.

\section{v}

Gogo

Oiseau frégate.

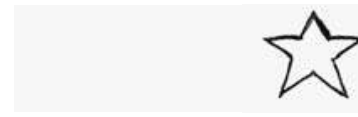

Fetū

Étoile.

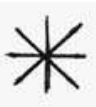

Alu'alu

Méduse.

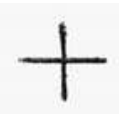

Toluse

Croix.

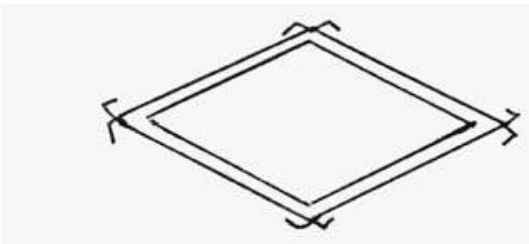

Malu

Abri, protection.

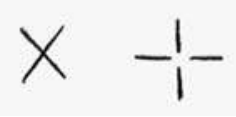

Ave'au

Étoile de mer. 
20. Selon ce principe structurel, «les formes que prennent les motifs et les figures sont celles qui impliquent le moins de modifications par rapport aux motifs avoisinants dont ils sont a priori distincts ". (Gell 2009 [1998] : 260) car leur forme renvoie assez directement à l'objet qu'elle dénote. Mais si leur degré d'iconicité est généralement supérieur à celui des vāega, le système de correspondance entre la forme des motifs et leur appellation est moins stable et le répertoire iconographique est lui-même en constante évolution. En effet, l'appellation des motifs décoratifs mamanu peut varier en fonction de leur positionnement dans une section ou selon l'idiosyncrasie de chaque maître tatoueur. À ce stade, c'est donc un «principe de moindre différence ${ }^{20}$ » qui semble gouverner la taille, le nombre et la transformation des motifs d'une œuvre à l'autre et d'un expert à l'autre.

La saillance visuelle des tatouages samoans ne tient pas à leur haut degré d'iconicité. Bien qu'il soit composé de motifs décoratifs et de formes intermédiaires nommées, ce type de tatouage standardisé ne semble pas destiné à livrer un code déchiffrable selon un système de correspondances entre images et appellations. Loin de contribuer à leur déchiffrement et à leur mémorisation (Severi 2007 [2004]), l'ordonnancement des images et des indices visuels en séquences participe plutôt d'un dispositif de brouillage cognitif, d'autant que les propriétés perceptives de l'œuvre, sa structure et ses motifs décoratifs ne sont pas associés à des pratiques d'énonciation qui rendraient leur forme particulièrement signifiante et faciliteraient le stockage en mémoire sémantique. En ce sens, l'idée énoncée par Philippe Descola (2006: 172) selon laquelle c'est «l'agencement qui fait l'agence», plutôt que l'inverse, semble tout à fait applicable au tatouage samoan: pe'a et malu résultent d'un processus d'enchâssement de motifs dans les sections et de sections dans l'œuvre finale aboutissant à une image complexe qui se présente comme un véritable piège à penser (Gell 2009 [1998] : 29).

Aussi, pour comprendre le mode d'encodage de ces images tatouées, on peut raisonnablement penser qu'il renvoie au «système de représentation perceptive» mis en évidence en psychologie cognitive par Endel Tulving et Daniel Schacter (1990) pour désigner un type de mémoire implicite présémantique spécialement dédié à la description structurale des objets. La notion de prototype (dont Alfred Gell a utilisé la fécondité en s'inspirant d'Eleanor Rosch [1973]) possède également une valeur heuristique indéniable tant du point de vue créatif que cognitif puisqu'elle permet notamment de comprendre comment la mémorisation d'un artefact aussi complexe peut aboutir à la formation d'une image mentale partagée. Les processus mémoriels et génératifs de l'image rituelle dépendent donc d'un principe de segmentation du corps qui rend possible la mémorisation du prototype tout en remplissant les conditions minimales pour que l'œuvre soit visuellement et rituellement efficace. II est, à ce titre, tout à fait significatif de constater que ce prototype est scrupuleusement respecté par des tufuga issus de familles différentes (su'a ou tulou'ena) à des époques différentes. Autrement dit, il est partagé par des praticiens qui n'ont pu avoir de contacts entre eux, ni «verticalement» dans leur généalogie de transmission, ni «horizontalement » durant leur apprentissage.

\section{"Articité" et variations individuelles dans l'ornementation}

En tant qu'exécutant, le tufuga ta tatau ne dispose que d'une très étroite marge de manœuvre pour singulariser ses productions: il est contraint par l'architecture du prototype et du rituel, mais il est aussi 
contraint socialement par le risque d'être qualifié d'orgueilleux (fia mimita) ou de prétentieux (fiapoto ${ }^{\mathbf{2 1}}$ ) car la réalisation d'un tatouage s'inscrit dans un ensemble de représentations culturelles valorisant l'humilité (agamaualalo). De fait, le lien entre l'image et son producteur reste très difficile à établir, de sorte que les maîtres tatoueurs les plus expérimentés ne sont pas toujours en mesure de reconnaître l'œuvre d'un confrère. Certains signent littéralement leurs travaux en piquant la partie externe de la jambe d'un motif distinctif (fig. 4 - [21]). Parmi les tufuga de la famille Sulu'ape, distinguer l'œuvre du père de celle de l'un de ses fils est une tâche quasi impossible, à moins d'avoir assisté à l'opération ou de connaître la signature respective de chaque membre de la famille (lorsque celle-ci existe). Outre cette pratique de la signature, qui s'apparente ici à un autographe et vient contredire la dimension «allographique» standard des tatouages, certaines données récentes permettent, sinon d'inférer une agentivité individuelle, du moins d'identifier une intention artistique de la part des tatoueurs et d'attribuer ainsi un certain degré d'«articité » à leurs œuvres ${ }^{22}$.

Celle-ci se manifeste sur le plan de la créativité par la formation de nouveaux motifs et sur le plan de la virtuosité par l'optimisation de la symétrie générale de l'œuvre, la régularité de l'espacement entre les motifs, leur quantité ou encore le nombre de lignes et de formes géométriques qui composent une section vāega (fig. 6 à 8). On remarque également un phénomène historique d'enrichissement progressif du répertoire ornemental et d'intensification dans la décoration de certaines vāega. Les tatouages documentés remontant à la fin du XIX ${ }^{\mathrm{e}}$ siècle et jusque dans les années 1920 (fig. 9) présentent une faible quantité d'options décoratives ${ }^{23}$. La plupart des zones étaient alors soit ornées d'un même motif ou d'une hachure, soit dépourvues de décoration. En revanche, le corpus iconographique disponible depuis les années 1930 montre que ces mêmes zones, autrefois peu ou pas décorées, sont désormais systématiquement ornées et dotées d'une plus grande diversité de motifs.

Je donnerai de ce phénomène une interprétation inspirée des recherches d'André Leroi-Gourhan (1963 et 1964) et de Jacques Fontanille (1998). Du fait de la rythmique propre à chaque outil et de la spécificité du son produit par le percuteur sur les instruments à tatouer, le tatouage constitue un type de figuration dont le principe est essentiellement rythmique. La diversification du répertoire ornemental semble relever d'une sémantisation progressive des rythmes manuels. Autrement dit, elle s'explique non par une planification, une idée de motifs dans l'esprit du tufuga finalement matérialisée dans le tatouage, mais plutôt, à partir de rythmes simples et répétitifs, par un processus de «prise de formes» des tracés rythmiques puis de sélection a posteriori de formes iconiques ressemblantes pour les désigner. À travers ce phénomène d'«iconisation", «l'icône devient un cas particulier, surdéterminé des formants décoratifs» (Fontanille 1998: 4). Dans la pratique du tatouage, cela se traduit par l'expérimentation de nouvelles formes décoratives qui ne sont pas gouvernées par une intention figurative. Ce qui est recherché, c'est un motif ornemental qui permette d'accroître la saillance visuelle de l'œuvre. On constate ainsi, en observant les tufuga contemporains au quotidien, l'apparition de nouveaux mamanu par transformation de motifs préexistants, c'est-à-dire par addition ou
21. Les pe'a et malu réalisés avec un foisonnement ornemental sont généralement considérés comme des œuvres caractéristiques de tatoueurs en début de carrière.

22. J'emprunte le terme à Gérard Genette (2010 [1994-1997] : 753). Par "articité", il entend notamment le caractère intentionnel objectivement établi d'une œuvre.

23. Pour un corpus représentatif, voir Buck 1930 ; voir également la peau tannée d'un Samoan originaire de Tutuila commentée par Francina Forment et Madeleine Brilot (dir., 2004 : 80). 
page ci-contre de haut en bas

fig. 6

Reproductions de la partie dorsale du tatouage masculin. Dessins Sébastien Galliot.

fig. 7

Reproductions de la zone aso fa'aifo. Dessins Sébastien Galliot.

fig. 8

Reproductions de la zone lausae/tapulu. Dessins Sébastien Galliot. soustraction de sections géométriques. La forme est déterminée à la fois par la technique de remplissage des lignes et des formes intermédiaires qui entrent dans la composition des zones, mais aussi par la forme des outils, lesquels permettent en priorité des formes géométriques et angulaires. Ainsi, la transformation d'un fa'avae'ali ("comme les pieds d'un appuie-tête samoan ") en i'a (poisson) peut s'opérer comme l'illustre la figure ci-dessous. Relativement à la technique de tatouage samoane, cette interprétation permet d'expliquer trois phénomènes: 1) la diversification du répertoire ornemental par transformation des motifs selon un principe de moindre différence; 2) le faible degré d'iconicité de certains motifs; 3) la faible valeur symbolique des unités ornementales. En effet, certains motifs récents (fig. 5) comme le fa'atigi pusa (littéralement "comme une boîte vide") ou le fa'ataimane ("comme un diamant») témoignent assez clairement du fait que leur création ne résulte pas d'une intention de l'artiste de figurer tel ou tel objet mais de contraintes matérielles dues à la forme des outils et d'une sémantisation a posteriori à partir d'inférences visuelles.

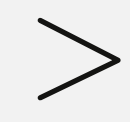

a. Forme initiale

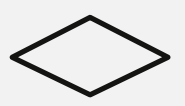

b. Forme intermédiaire

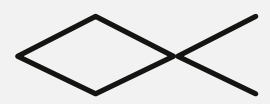

c. Forme transformée

\section{Conclusion}

Comme tout rite initiatique, le tatouage samoan est une action transformative, mais le fait que celle-ci soit exécutée par une classe d'experts technico-rituels rend la compréhension de sa transmission problématique car il institue d'emblée un écart cognitif entre agents et patients. La configuration relationnelle spécifique à ce rite en fait une forme culturelle assez singulière dans la mesure où sa transmission passe par la juxtaposition, dans un même rite, de dispositifs mnémoniques distincts. Ceci remet en question une idée répandue sur les rites initiatiques selon laquelle les acquis - qui sont soit de l'ordre de l'indicible car ils relèvent d'une expérience exceptionnelle, soit de l'ordre d'un savoir doctrinal secret - se manifestent dans la capacité des novices à devenir eux-mêmes initiateurs (La Fontaine 1977). Or, à Samoa, seuls les experts incarnent à la fois la connaissance des novices et celle des initiateurs.

Inscrit dans la peau, l'ensemble du répertoire ornemental est conservé sur le corps des patients pour une durée limitée et n'a pas vocation à être appris ni reproduit à partir de ce support vivant. Le droit de reproduction étant restreint à un petit nombre d'experts, la visibilité, l'omniprésence et la longévité des œuvres ne garantissent pas leur conservation. Autrement dit, le support matériel (le corps) et l'image tatouée ne s'imposent pas comme des supports mnémoniques cruciaux pour la transmission de l'œuvre et du rite mais jouent plutôt sur la motivation des futurs initiés et le choix éventuel d'un tatoueur que l'on jugera plus talentueux qu'un autre. Les différentes actions subies par les patients et le contexte relationnel, spatial, olfactif et sonore (douleur intense et prolongée suivie de massages quotidiens et d'une onction finale, odeur du sang, du curcuma, des colliers de fleurs, son des outils, chants occasionnels des participants) contribuent à la formation 

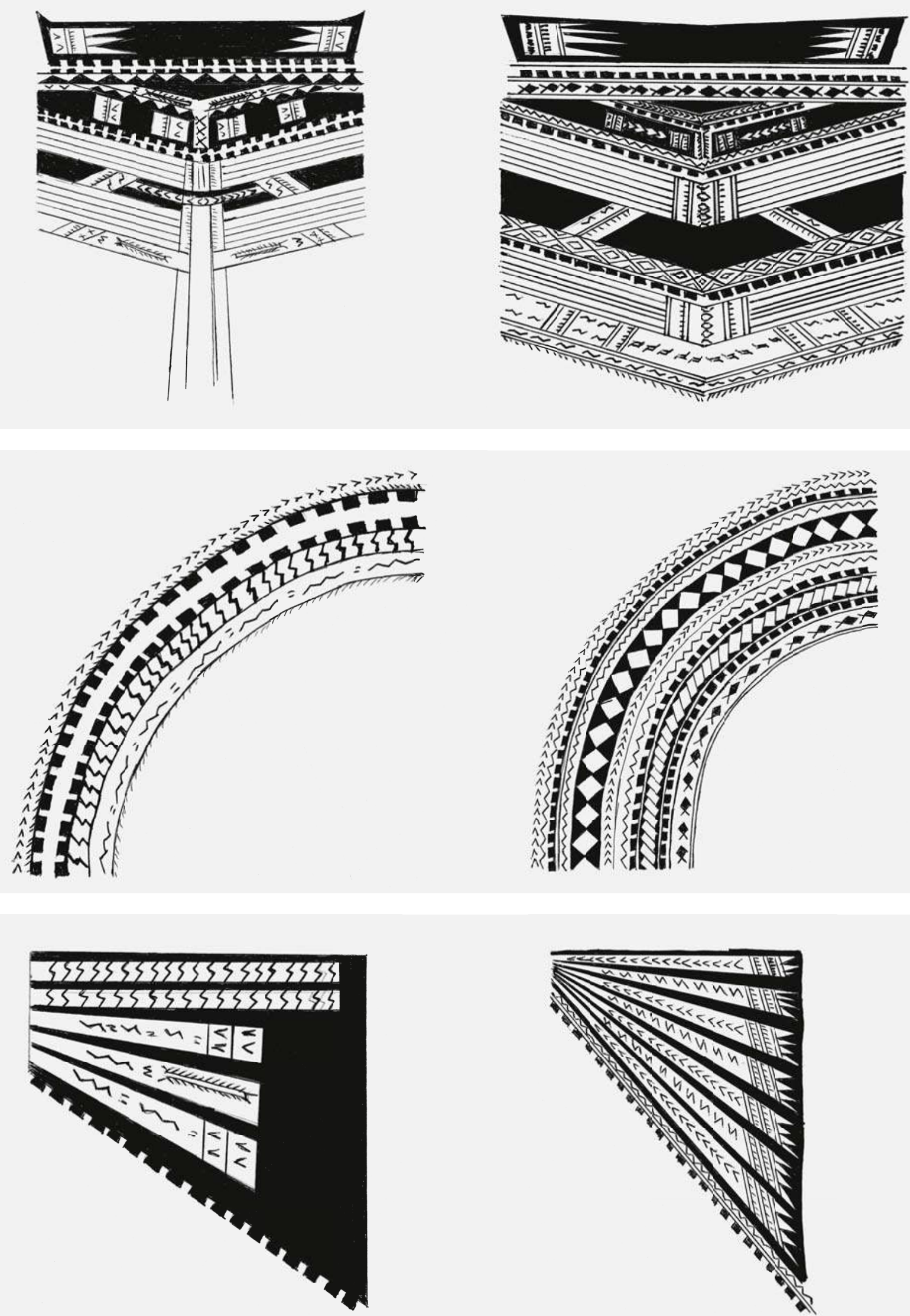


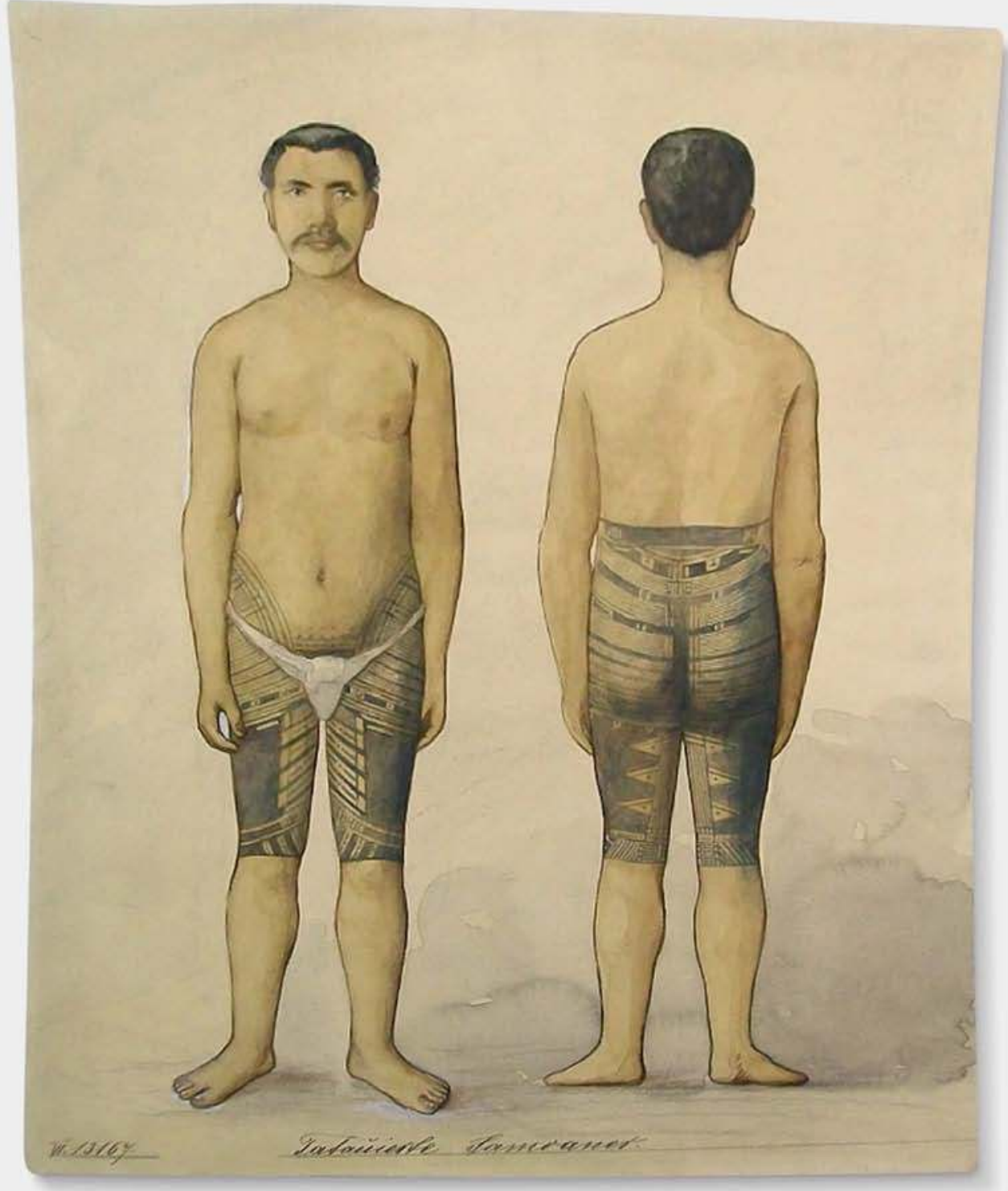


d'une mémoire autobiographique. Dans ce cas précis, ce type de mémoire n'est pas mobilisé pour conserver des détails sur les procédures rituelles mais pour garder un ensemble de sensations vécues en groupe ainsi qu'une vive trace mnésique du contexte et de l'identité des participants.

Du point de vue des agents du rituel, les contenus de savoirs à mémoriser ne concernent pas la tradition orale de la mythologie du tatouage ou la nomenclature des motifs ornementaux, pas plus que la mémorisation de commentaires exégétiques approfondis (Whitehouse 2000), mais sont de l'ordre des savoir-faire techniques incorporés (Mahias 2002: 99). Ce type de savoirs implicites et non verbaux est caractéristique des connaissances stockées en mémoire procédurale (Tulving 1972). Cette dernière conserve sous forme de scripts et de schémas d'actions les connaissances liées à la réalisation d'un tatouage. Le savoir procédural implicite du tatoueur et de ses assistants et apprentis s'acquiert par la répétition des gestes dans lesquels ils sont engagés de façon quasi quotidienne. II s'agit bien d'une mémoire partagée, procédurale, implicite, pré-sémantique, indissociable du geste et de l'action, qui revêt toutes les qualités d'une «protomémoire» (Candau 2005 [1996] : 77-78). Néanmoins, si une partie des gestes du tatoueur (comme l'appariement entre percussion et type d'outil) semble machinaux, la réalisation de l'œuvre nécessite toujours une grande concentration et les actes techniques commis ne sont pas tous inconscients et indicibles ${ }^{\mathbf{2 4}}$. Cette expertise technique liée au caractère routinier des actions rituelles va de pair avec le développement d'une forme d'orthodoxie qui n'est pas doctrinale et transmise verbalement (Whitehouse 2000 et 2004) mais opératoire et apprise par corps. Ainsi, détaché du système religieux et n'agissant pas comme support de la croyance, le rite de tatouage produit un cadre social pour la mémoire professionnelle des experts et autobiographique des patients.

Centre de recherche et de documentation sur l'Océanie s.galliot1@gmail.com
24. L'expert peut formuler assez clairement les différentes étapes de la conception de ses outils et de certaines actions rituelles et redessiner avec précision une œuvre qu'il ne réalise généralement qu'à l'aide d'un percuteur et d'un outil à tatouer.

\section{ci-contre}

fig. 9

Samoan tatoué. Aquarelle réalisée par Her Fresenius sous la supervision de Felix von Luschan à l'occasion du passage d'une troupe de Samoans au Panoptikum de Berlin en 1895. (C) Photo: Musée ethnologique des musées nationaux de Berlin - Patrimoine culturel prussien. remerciements

Mes remerciements vont à Laurent Berger, Jean-Pierre Warnier, Pierre Lemonnier et Vincent Hirtzel pour leurs relectures attentives et leurs précieux commentaires. 
Bibliographie

\section{Abercromby, John}

1891 "Samoan Stories", Folklore II (4) : 455-467.

\section{Balfet, Hélène}

1975 "Technologie», in Robert Cresswell (éd.), Éléments d'ethnologie, vol. II, Six approches. Paris, Armand Colin ("Collection U sociologie") : 44-79.

\section{Barth, Fredrik}

1975 Ritual and Knowledge among the Baktaman of New Guinea. Oslo et New Haven, UniversitetsforlagetYale University Press.

\section{Bonnet, Claude}

1989 "La perception visuelle des formes ", in Claude Bonnet, Rodolphe Ghiglione et Jean-François Richard (éd.), Traité de psychologie cognitive, vol. I, Perception, Langage. Paris, Dunod ("Sciences humaines Dunod »): 1-82.

\section{Brunt, Peter}

2005 «The temptation of Brother Anthony: Decolonization and the tattooing of Tony Fomison ", in Thomas, Cole et Douglas (éd.) 2005: 123-144.

\section{Buck, Peter}

\section{(Te Rangi Hiroa)}

1930 Samoan Material Culture. Honolulu, The Museum («Bernice P. Bishop Museum Bulletin» 75).

\section{Candau, Joël}

2005 [1996] Anthropologie de la mémoire. Paris, Armand Colin («Cursus. Série Sociologie»).

\section{Chamoux, Marie-Noëlle}

1978 "La transmission des savoirfaire: un objet pour l'ethnologie des techniques? ", Techniques et culture 3: 46-83.

\section{Coupaye, Ludovic}

2013 Growing Artefacts, Displaying Relationships. Yams, Art and Technology Amongst the Nyamikum Abelam of Papua New Guinea, vol. II, Materials and Mediations: People and Things in a World of Movement. Oxford et New York, Berghahn Books.

Le tatouage samoan et ses agents. Images, mémoire et actions rituelles

Par Sébastien Galliot

\section{Csordas, Thomas J. (éd.)}

1994 Embodiment and Experience. The Existantial Ground of Culture and Self. Cambridge, Cambridge University Press ("Cambridge studies in medical anthropology » 2).

\section{Descola, Philippe}

2006 "La fabrique des images", Anthropologie et Société XXX (3): 167-182.

\section{Faure, Sylvia}

2000 Apprendre par corps. Socio-anthropologie des techniques de danse. Paris, La Dispute.

\section{Fontanille, Jacques}

1998 "Décoratif, iconicité et écriture. Geste, rythme et figurativité: à propos de la poterie berbère", Visio III (2) ("Histoire de l'art et sémiotique. Semiotics and art history "): 33-46

\section{Forment, Francina et Brilot, Madeleine (dir.)}

2004 Tatu-Tattoo! Bruxelles et Anvers, Musées royaux d'Art et d'Histoire-Fonds Mercator.

\section{Fraser, John}

1896 "Some folk-songs and myths from Samoa ", Journal of the Polynesian Society V (3) : 171-182.

\section{Friedman, Johnattan}

1975 «Tribes, States, and Transformations ", in Maurice Bloch, (éd.), Marxist Analyses in Social Anthropology. Londres, Malaby Press: 161-202.

\section{Galliot, Sébastien}

2010 Pe'a et Malu. Le tatouage à Samoa (1722-2010): technique et culture dans une société de Polynésie occidentale en mutation, thèse,

École des hautes études en sciences sociales.

À paraître "Ritual efficacy in the making. Materiality, actions, substances in the Samoan tattooing ", Journal of Material Culture.

\section{Gell, Alfred}

1993 Wrapping in Images. Oxford et New York, Clarendon Press-Oxford University Press ( Oxford studies in social and cultural anthropology").

2009 [1998] L'Art et ses agents. Une théorie anthropologique, trad. de l'anglais par Sophie Renaut et Olivier Renaut. Dijon, Les Presses du réel ("Fabula»)

\section{Genette, Gérard}

2010 [1994-1997] L'CEuvre de l'art Paris, Seuil ("Poétique»).

\section{Goldman, Irving}

1970 Ancient Polynesian Society. Chicago, University of Chicago Press.

\section{Grand, Simone}

2007 Tahua'a, tohunga, kahuna. Le monde polynésien des soins traditionnels. Pirae, Au Vent des îles.

\section{Handy, Craighill E.S. et Handy, Willowdean \\ Chaterson}

1924 Samoan House Building, Cooking, and Tattooing. Honolulu, The Museum («Bernice P. Bishop Museum Bulletin » 15)

\section{Holmes, Lowell D.}

1957 "Ta'u, stability and change in a Samoan village ", Journal of the Polynesian Society, LXVI (3): 301-338.

\section{Houseman, Michael}

1993 "The interactive basis of ritual effectiveness in a male initiation rite", in Pascal Boyer (éd.), Cognitive Aspects of Religious Symbolism. Cambridge et New York, Cambridge University Press: 207-224.

1999 «Quelques configurations relationnelles de la douleur", in Françoise Héritier (dir.), De la violence, t. II. Paris, Odile Jacob: 77-112.

\section{Houseman, Michael et Severi, Carlo}

1994 Naven ou Le donner à voir. Essai d'interprétation de l'action rituelle. Paris, CNRS Éditions-Maison des sciences de l'homme ("Chemins de l'ethnologie").

\section{Hutchins, Edwin}

2005 «Material anchors for conceptual blends ", Journal of Pragmatics 37 : 1555-1577.

Julien, Marie-Pierre et Warnier, Jean-Pierre (dir.)

1999 Approches de la culture matérielle. Corps à corps avec l'objet. Paris, L'Harmattan (“Connaissance des hommes").

\section{Keesing, Felix M.}

1937 "The taupo system of Samoa. A study of institutionnal change", Oceania, VIII (1) : 1-14.

\section{Krämer, Augustin}

1994-1995 [1902-1903]

The Samoan Islands. An Outline of a Monograph With Particular Consideration of German Samoa. Auckland et Honolulu, Polynesian Press-University of Hawai'i Press.

\section{Küchler, Susanne}

2002 Malanggan: Art, Memory and Sacrifice. Oxford et New York, Berg («Materializing culture»).

\section{Kuwahara, Makiko}

2005 Tattoo. An anthropology. Oxford et New York, Berg.

\section{La Fontaine, Jean}

1977 "The power of rights", Man (nouvelle série) XII (3-4): 421-437.

\section{Lave, Jean}

\section{et Wenger, Etienne}

1991 Situated Learning: Legitimate Peripheral Participation, Cambridge: Cambridge University Press, 


\section{Lemonnier, Pierre}

2005 "Mythiques chaînes opératoires ", Techniques et cultures 43-44:25-43.

2010 "Vingt fois le "faire" est repensé. Ou quand l'action technique mobilise l'imaginaire ", in Françoise Cousin et Christian Pelras (dir.), Matières, manières et sociétés. Hommage à Hélène Balfet. Aix-en-Provence, Publications de I'Université de Provence: 295-302.

2012 "Technology ", in Nicholas Thieberger (éd.), The Oxford Handbook of Linguistic Fieldwork. Oxford, Oxford University Press : 298-316.

\section{Leroi-Gourhan, André}

1963 Le Geste et la Parole, vol. I, Technique et Langage. Paris, Albin Michel ("Sciences d'aujourd'hui »).

1964 Le Geste et la Parole, vol. II, La Mémoire et les Rythmes.

Paris, Albin Michel ("Sciences d'aujourd'hui »).

\section{Mahias, Marie-Claude}

2002 Le Barattage du monde. Essais d'anthropologie des techniques en Inde. Paris, Éditions de la Maison des sciences de l'homme ("Archéologie expérimentale et ethnographie des techniques » 6)

\section{Malinowski, Bronisław}

1935 Coral Garden and their Magic. A Study of the Method of Tiling the Soil and of Agricultural Rites in the Trobriands Islands. Londres, Allen \& Unwin, 2 vol.

\section{Mallon, Sean}

2005 «Samoan Tatau as global practice ", in Thomas, Cole et Douglas (éd.) 2005: 145-170.

\section{Mangos, Therese et Utanga, John}

2011 Patterns of the Past. Tattoo Revival in the Cook /slands. Auckland, Punarua Productions.

\section{Marquardt, Carl}

1984 [1899] The Tattooing of Both Sexes in Samoa, trad. de l'allemand par Sibyl Ferner. Papakura, McMillan.

\section{Martinelli, Bruno}

1997 "Sous le regard de l'apprenti. Paliers de savoir et d'insertion chez les forgerons moose du Yatenga (Burkina Faso) ", Techniques et Culture ("Accès aux savoir d'autrui ", Marie-Noëlle Chamoux [dir.]) 28: 9-47.

\section{McCauley, Robert N. et Lawson, E. Thomas}

2002 Bringing Ritual to Mind. Psychological Foundations of Cultural Forms. Cambridge et New York, Cambridge University Press.

\section{McGrevy, Noel Lawrence}

1973 O Le Ta Tatau: An Examination of Certain Aspects of Samoan Tattooing to the Present, thèse, université d'Hawaï.

\section{Milner, George B.}

1969 «Siamese twins, birds and the double helix ", Man (nouvelle série), IV (1) : 5-23.

\section{Mitchell, Jon P.}

2006 "Performance", in Christopher Y. Tilley et al. (éd.), Handbook of Material Culture. Thousand Oaks, Sage : 384-401.

\section{Morphy, Howard}

1991 Ancestral Connections. Art and an Aboriginal System of Knowledge. Chicago, University of Chicago Press.

\section{Olsen, Bjørnar}

2010 In Defense of Things. Archeology and the Ontology of Objects. Plymouth, AltaMira Press.

\section{Rosch, Eleanor}

1973 "Natural categories», Cognitive Psychology IV (3) : 328-350.

\section{Sahlins, Marshall}

1958 Social Stratification in Polynesia. Seattle, University of Washington Press (« Monographs of the American Ethnological Society " 29).

\section{Severi, Carlo}

2007 [2004] Le Principe de

la chimère. Une anthropologie de la mémoire. Paris, Rue d'Ulm/Presses de l'École normale supérieure-

musée du quai Branly (" Esthetica»).

\section{Stair, John B.}

1983 [1897] Old Samoa or Flotsam and Jetsam from the Pacific Ocean. Papakura, McMillan.

\section{Tcherkézoff, Serge}

2003 Faa-Samoa, une identité polynésienne. Économie, politique, sexualité. L'anthropologie comme dialogue culturel. Paris, L'Harmattan ("Connaissance des hommes").

\section{Te Awekotuku, Ngahuia} et Waimarie Nikora, Linda

2010 [2007] Mau Moko. Le monde du tatouage maori, trad. de l'anglais par Marc Orlando. Pirae, Au Vent des îles ("Culture Pacifique»).

\section{Thomas, Nicholas}

1990 Marquesan Societies. Inequality and Political Transformation in East Polynesia. Oxford et New York, Clarendon Press-Oxford University Press.

\section{Thomas, Nicholas,} Cole, Anna et Douglas, Bronwen (éd.)

2005 Tattoo. Bodies, Art, and Exchange in the Pacific and the West. Durham, Duke University Press ("Objects/histories").

\section{Tulving, Endel}

1972 "Episodic and semantic memory ", in Endel Tulving et Wayne Donaldson (éd.), Organization of Memory. New York et Londres, Academic Press: 381-403.

\section{Tulving, Endel et} Schacter, Daniel. L.

1990 "Priming and human memory systems ", Science CCXLVII (4940): 301-306.

\section{Warnier, Jean-Pierre}

2009 [2007] Régner au Cameroun. Le Roi-Pot. Paris, Karthala

(«Recherches internationales»).

\section{Whitehouse, Harvey}

2000 Arguments and lcons. Divergent Modes of Religiosity. Oxford et New York, Oxford University Press.

2004 Modes of Religiosity. A Cognitive Theory of Religious Transmission. Walnut Creek, AltaMira Press.

\section{Wilkes, Charles}

1856 [1842] Narrative of the United States Exploring Expedition during the years 1838, 1839, 1840, 1841, 1842, vol. II. New York, Putnam \& Co. 